\title{
Arikan Meets Shannon: Polar Codes with Near-Optimal Convergence to Channel Capacity
}

\author{
Venkatesan Guruswami \\ Carnegie Mellon University \\ Pittsburgh, PA, USA \\ venkatg@cs.cmu.edu
}

\author{
Andrii Riazanov \\ Carnegie Mellon University \\ Pittsburgh, PA, USA \\ riazanov@cs.cmu.edu
}

\author{
Min Ye \\ Tsinghua-Berkeley Shenzhen Institute \\ Shenzhen, China \\ yeemmi@gmail.com
}

\begin{abstract}
Let $W$ be a binary-input memoryless symmetric (BMS) channel with Shannon capacity $I(W)$ and fix any $\alpha>0$. We construct, for any sufficiently small $\delta>0$, binary linear codes of block length $O\left(1 / \delta^{2+\alpha}\right)$ and rate $I(W)-\delta$ that enable reliable communication on $W$ with quasi-linear time encoding and decoding. Shannon's noisy coding theorem established the existence of such codes (without efficient constructions or decoding) with block length $O\left(1 / \delta^{2}\right)$. This quadratic dependence on the gap $\delta$ to capacity is known to be the best possible. Our result thus yields a constructive version of Shannon's theorem with near-optimal convergence to capacity as a function of the block length. This resolves a central theoretical challenge associated with the attainment of Shannon capacity. Previously such a result was only known for the binary erasure channel.

Our codes are a variant of Arıkan's polar codes based on multiple carefully constructed local kernels, one for each intermediate channel that arises in the decoding. A crucial ingredient in the analysis is a strong converse of the noisy coding theorem when communicating using random linear codes on arbitrary BMS channels. Our converse theorem shows extreme unpredictability of even a single message bit for random coding at rates slightly above capacity.
\end{abstract}

\section{CCS CONCEPTS}

- Theory of computation $\rightarrow$ Error-correcting codes; $\bullet$ Mathematics of computing $\rightarrow$ Coding theory.

\section{KEYWORDS}

polar codes, capacity-achieving codes, information theory

\section{ACM Reference Format:}

Venkatesan Guruswami, Andrii Riazanov, and Min Ye. 2020. Arikan Meets Shannon: Polar Codes with Near-Optimal Convergence to Channel Capacity. In Proceedings of the 52nd Annual ACM SIGACT Symposium on Theory of Computing (STOC '20), fune 22-26, 2020, Chicago, IL, USA. ACM, New York, NY, USA, 13 pages. https://doi.org/10.1145/3357713.3384323

Permission to make digital or hard copies of all or part of this work for personal or classroom use is granted without fee provided that copies are not made or distributed for profit or commercial advantage and that copies bear this notice and the full citation on the first page. Copyrights for components of this work owned by others than the author(s) must be honored. Abstracting with credit is permitted. To copy otherwise, or republish, to post on servers or to redistribute to lists, requires prior specific permission and/or a fee. Request permissions from permissions@acm.org.

STOC '20, June 22-26, 2020, Chicago, IL, USA

(c) 2020 Copyright held by the owner/author(s). Publication rights licensed to ACM. ACM ISBN 978-1-4503-6979-4/20/06 . \$ \$15.00

https://doi.org/10.1145/3357713.3384323

\section{INTRODUCTION}

We construct binary linear codes that achieve the Shannon capacity of the binary symmetric channel, and indeed any binary-input memoryless symmetric (BMS) channel, with a near-optimal scaling between the code length and the gap to capacity. Further, our codes have efficient (quasi-linear time) encoding and decoding algorithms. Let us now describe the context of our result and its precise statement in more detail.

The binary symmetric channel (BSC) is one of the most fundamental and well-studied noise models in coding theory. The BSC with crossover probability $p \in(0,1 / 2)\left(\mathrm{BSC}_{p}\right)$ flips each transmitted bit independently with probability $p$. By Shannon's seminal noisy coding theorem [25], we know that the capacity of $\mathrm{BSC}_{p}$ is $1-h(p)$, where $h(\cdot)$ is the binary entropy function. This means that reliable communication over $\mathrm{BSC}_{p}$ is possible at information rates approaching $1-h(p)$, and at rates above $1-h(p)$ this is not possible. More precisely, for any $\delta>0$, there exist codes of rate $1-h(p)-\delta$ using which one can achieve miscommunication probability at most $2^{-\Omega\left(\delta^{2} n\right)}$ where $n$ is the block length of the code. In fact, random linear codes under maximum likelihood decoding offer this guarantee with high probability. Thus Shannon's theorem implies the existence of codes of block length $O\left(1 / \delta^{2}\right)$ that can achieve small error probability on $\mathrm{BSC}_{p}$ at rates within $\delta$ of capacity. Conversely, by several classical results $[23,26,27,31]$, we know that the block length has to be at least $\Omega\left(1 / \delta^{2}\right)$ in order to approach capacity within $\delta$.

Shannon's theorem is based on the probabilistic method and does not describe the codes that approach capacity or give efficient algorithms to decode them from errors caused by $\mathrm{BSC}_{p}$. Thus the codes with rates $1-h(p)-\delta$ take at least time exponential in $1 / \delta^{2}$ to construct as well as decode. This is also true for concatenated coding schemes [9] as the inner codes have to be decoded by bruteforce, and either have to also be found by a brute-force search or allowed to vary over an exponentially large ensemble (leading to exponentially large block length).

The theoretical challenge of constructing codes of rate $1-h(p)-\delta$ with construction/decoding complexity scaling polynomially in $1 / \delta$ in fact remained wide open for a long time. Finally, around 2013, two independent works $[14,15]$ gave an effective finite-length analysis of Arıkan's remarkable polar codes construction [1]. (Arıkan's original analysis, as well as follow-ups like [2], proved convergence to capacity as the block length grew to infinity but did not quantify the speed of convergence.) Based on this, a construction of polar codes with block length, construction, and decoding complexity all bounded by a polynomial in $1 / \delta$ to capacity was obtained in 
$[14,15]$. The result also applies to any BMS channel, not just the BSC.

If the block length of the code scales as $O\left(1 / \delta^{\mu}\right)$ as a function of the gap $\delta$ to capacity, we say that $\mu$ is the scaling exponent. The above results established that the scaling exponent of polar codes is finite. It is worth pointing out that polar codes are the only known efficiently decodable capacity-achieving family proven to have a finite scaling exponent. The work [14] did not give an explicit upper bound on the scaling exponent of polar codes, whereas [15] showed the bound $\mu \leq 6$. Following some improvements in [13, 20], the current best known upper bound on $\mu$ for the BSC (and any BMS channel) is 4.714 .

Note that random linear codes have optimal scaling exponent 2. The above results thus raise the intriguing challenge of constructing codes with scaling exponent close to 2 , a goal we could not even dream of till the recent successes of polar codes.

Arıkan's original polar coding construction is based on a large tensor power of a simple $2 \times 2$ matrix, which is called the kernel of the construction. For this construction, it was shown in [15] that the scaling exponent $\mu$ for Arıkan's original polar code construction is lower bounded by 3.579, even for the simple binary erasure channel. Given this limitation, one approach to improve $\mu$ is to consider polar codes based on $\ell \times \ell$ kernels for larger $\ell$. However, better upper bounds on the scaling exponent of polar codes based on larger kernels have not been established except for the simple case of the binary erasure channel (BEC). ${ }^{1}$ For the BEC, using large kernels, polar codes with scaling exponent $2+\alpha$ for any desired $\alpha>0$ were given in the very nice paper [7] which spurred our work. (We will discuss this and other related works in more detail in Sections 2.2-2.3.)

Our main result in this work is a polynomial time construction of polar codes based on large kernels that approach the optimal scaling exponent of 2 for every BMS channel. Specifically, for any desired $\alpha>0$, by picking sufficiently large kernels (as a function of $\alpha$ ), the block length $N$ can be made as small as $O_{\alpha}\left(1 / \delta^{2+\alpha}\right)$ for codes of rate $I(W)-\delta$ (the notation $O_{\alpha}(\cdot)$ hides a constant that depends only on $\alpha$ ). The encoding and decoding complexity will be quasi-linear in $N$, and thus can also have a near-quadratic growth with $1 / \delta$.

Theorem 1.1 (MAIN). Let $W$ be an arbitrary BMS channel with Shannon capacity $I(W)$. For any $c>0$ and arbitrarily small $\alpha>0$, if we choose a constant $\ell \geq \ell_{0}(\alpha)$ to be a power of 2 , then there is a code $C$ generated by the polar coding construction using kernels of size $\ell \times \ell$ such that the following four properties hold when the code length $N$ grows: (1) the code construction has $N^{O_{\alpha}(1)}$ complexity; (2) both encoding and decoding have $O_{\alpha}(N \log N)$ complexity; (3) rate of $C$ is $I(W)-N^{-1 / 2+(c+6) \alpha}$; (4) decoding error probability is bounded by $O_{\alpha}\left(\log N / N^{c}\right)$ when $C$ is used for channel coding over $W$.

The above "constructivizes" the quantitative finite-length version of Shannon's theorem with a small $\alpha$ slack in the speed of convergence to capacity. The lower bound on the constant $\ell$ can be chosen as $\ell_{0}(\alpha)=\exp \left(\alpha^{-1.01}\right)$. Note that a similar lower bound on $\ell$ also appears in the aforementioned result for the BEC from [7].

\footnotetext{
${ }^{1}$ Polar codes based on $\ell \times \ell$ kernels have much larger block length $\ell^{t}$ compared to $2^{t}$ for the $2 \times 2$ case. So to get an improvement in $\mu$, one has to compensate for the increasing block length via better bounds on the local behavior of the kernel.
}

We restrict our attention to binary-input symmetric channels in this paper. Wang and Duursma extended the results of Theorem 1.1 to any discrete memoryless channels (possibly non-binary and asymmetric) in [30]. They also presented a tighter analysis of decoding error probability, which allowed to drive it down to inverse exponential in $N\left(\exp \left(-N^{O(\alpha)}\right)\right.$ in the context of Theorem 1.1), as opposed to inverse polynomial decoding error probability, which we achieve in this paper. However, this was done at a cost of losing the polynomial-time construction complexity of the code. In the full version of this paper, we show how to combine the analysis from [30] with our approach of constructing the code to achieve both the poly-time construction and exponentially small decoding error probability simultaneously.

\section{OVERVIEW OF CONSTRUCTION AND ANALYSIS}

In order to better explain our work and situate it in the rich backdrop of related works on polar codes, we begin with some context and background on the phenomenon of channel polarization that lies at the heart of Arrkan's polar coding approach.

\subsection{Channel Transforms, Entropy Polarization, and Polar Codes}

Consider an arbitrary binary-input memoryless symmetric (BMS) ${ }^{2}$ channel $W:\{0,1\} \rightarrow \mathcal{Y}$, and an $\ell \times \ell$ invertible binary matrix $K$ (referred to as the kernel). Suppose that we are transmitting a binary vector $\boldsymbol{U}=\left(U_{1}, U_{2}, \ldots, U_{\ell}\right)$ uniformly chosen from $\{0,1\}^{\ell}$ in the following way: first, it is transformed into $\boldsymbol{X}=\boldsymbol{U} K$, which is then transmitted through $\ell$ copies of the channel $W$ to get the output $\boldsymbol{Y}=W^{\ell}(\boldsymbol{X}) \in \boldsymbol{y}^{\ell}$.

Now imagine decoding the input bits $U_{i}$ successively in the order of increasing $i$. This naturally leads to a binary-input channel $W_{i}$ : $\{0,1\} \rightarrow \mathcal{Y}^{\ell} \times\{0,1\}^{i-1}$, for each $i \in[\ell]$, which is the channel "seen" by the bit $U_{i}$ when all the previous bits $\boldsymbol{U}_{<i}$ and all the channel outputs $\boldsymbol{Y} \in \mathcal{Y}^{\ell}$ are known. Formally, the transition probabilities of this channel are

$$
W_{i}\left(\boldsymbol{Y}, \boldsymbol{U}_{<i} \mid U_{i}\right)=\frac{1}{2^{\ell-i}} \sum_{\boldsymbol{V} \in\{0,1\}^{\ell-i}} W^{\ell}\left(\boldsymbol{Y} \mid\left(\boldsymbol{U}_{<i}, U_{i}, \boldsymbol{V}\right) K\right),
$$

where $\boldsymbol{U}_{<i} \in\{0,1\}^{i-1}$ are the first $(i-1)$ bits of $\boldsymbol{U}$, and the sum is over all possible values $\boldsymbol{V} \in\{0,1\}^{\ell-i}$ that the last $(\ell-i)$ bits of $\boldsymbol{U}$ can take. In this paper we will address the channel $W_{i}$ as "Arıkan's bit-channel of $W$ with respect to $K . "$

A polarization transform associated with the kernel $K$ is then defined as a transformation that maps $\ell$ copies of the channel $W$ to the channels $W_{1}, W_{2}, \ldots, W_{\ell}$. For a BMS channel $W$, we define $H(W)$ as the conditional entropy of the channel input random variable given the channel output random variable when the channel input has uniform distribution. Since $K$ is invertible, a direct implication of the chain rule for entropy gives entropy conservation property,

\footnotetext{
${ }^{2}$ We say that a channel $W:\{0,1\} \rightarrow \mathcal{Y}$ is a BMS channel if there is a permutation $\pi$ on the output alphabet $\mathcal{Y}$ satisfying i) $\pi^{-1}=\pi$ and ii) $W(y \mid 1)=W(\pi(y) \mid 0)$ for all $y \in \mathcal{Y}$.
} 
which is

$$
\begin{aligned}
\ell \cdot H(W)=H(\boldsymbol{X} \mid \boldsymbol{Y})=H(\boldsymbol{U} \mid \boldsymbol{Y}) & =\sum_{i=1}^{\ell} H\left(U_{i} \mid \boldsymbol{U}_{<i}, \boldsymbol{Y}\right) \\
& =\sum_{i=1}^{\ell} H\left(W_{i}\right) .
\end{aligned}
$$

If $K$ is invertible and is not upper-triangular under any column permutation (which we refer to as a mixing matrix), then the bitchannels $W_{1}, W_{2}, \ldots, W_{\ell}$ start polarizing-some of them become better than $W$ (have smaller entropy), and some become worse. The standard approach is then to recursively apply the polarization transform of $K$ to these bit-channels. This naturally leads to an $\ell$-ary tree of bit-channels. The $t^{\text {th }}$ level of the tree corresponds to the linear transformation $K^{\otimes t}$, the $t$-fold Kronecker product of $K .^{3}$

In his landmark paper [1], Arıkan proved that when $K=\left(\begin{array}{ll}1 & 0 \\ 1 & 1\end{array}\right)$, at the $t^{\text {th }}$ level, all but a $o(1)$ fraction of the channels (as $\left.t \rightarrow \infty\right)$ are either almost noiseless (have tiny entropy) or completely useless (have entropy very close to 1 ). To get capacity-achieving codes from polarization, the idea is to use the almost-noiseless channels, which will constitute $\approx I(W)$ fraction by conservation of entropy, to carry the message bits, and "freeze" the bits in the remaining positions to pre-determined values (eg. all $0 \mathrm{~s}$ ). Thus the generator matrix of the code will consist of those rows of $K^{\otimes t}$ that correspond to the almost-noiseless positions. Arıkan presented a successive cancellation (SC) decoder and proved that it can be implemented using $O(N \log N)$ operations where $N=\ell^{t}$ is the code length, thanks to the nice recursive structure of $K^{\otimes t}$.

For the parameters of the code, if one shows that at most $\delta_{t}$ fraction of the channels at the $t^{\text {th }}$ level have entropies in the range $\left(\zeta_{t}, 1-\zeta_{t}\right)$, then one (roughly) gets codes of length $2^{t}$, rate $I(W)-$ $\delta_{t}-\zeta_{t}$, for which the SC decoder achieves decoding error probability $\zeta_{t} \ell^{t}$ for noise caused by $W$ (see, for example [5, Theorem A.3]). Thus, one needs $\zeta_{t}$ sub-exponentially small in $t$ (i.e., at most $\left.\exp (-\omega(t))\right)$ to achieve good decoding error. For Arıkan's original $2 \times 2$ kernel, this was shown in [2]. Korada, Sasoglu and Urbanke extended the analysis to arbitrary $\ell \times \ell$ mixing matrices over the binary field [17], and Mori and Tanaka established a similar claim over all finite fields [21].

The fraction $\delta_{t}$ of unpolarized channels (whose entropies fail to be sub-exponentially close to 0 or 1 ) governs the gap to capacity of polar codes. The above works established that $\lim _{t \rightarrow \infty} \delta_{t}=0$, and thus polar codes achieve capacity asymptotically as the block length grows to infinity. However, they did not provide any bounds on the speed at which $\delta_{t} \rightarrow 0$ as a function $t$, much less quantify a scaling exponent. Note that one would need to show $\delta_{t} \leq O\left(\ell^{-t / \mu}\right)$ to establish a scaling exponent of $\mu$, since the code length is $\ell^{t}$.

\subsection{Scaling Exponents: Prior Work}

For Arıkan's original kernel $\left(\begin{array}{ll}1 & 0 \\ 1 & 1\end{array}\right)$, two independent works [14, 15] proved that $\delta_{t}$ drops to 0 exponentially fast in $t$. This proved that Arıkan's polar codes have finite scaling exponent (i.e., converge to capacity polynomially fast in the block length), the first codes

\footnotetext{
${ }^{3}$ Actually, the analysis is more convenient if one applies a bit-reversal permutation of the $U_{i}$ 's, and indeed we do so also in this paper, but this is not important for our current discussion.
}

with this important feature. Blasiok et al generalized this result significantly [5], proving that the entire class of polar codes, based on arbitrary mixing matrices over any prime field as kernels, has finite scaling exponent.

For concrete upper bounds on the scaling exponent, the work of Hassani, Alishahi, and Urbanke [15] had proved $\mu \leq 6$ for Arıkan's original kernel. This was improved to $\mu \leq 5.702$ in [13]. Mondelli, Hassani, and Urbanke [20] showed that $\mu \leq 4.714$ for any BMS channel $W$, and showed a better upper bound $\mu \leq 3.639$ for the case when $W$ is a binary erasure channel (BEC). A lower bound $\mu \geq 3.579$ appears in [15] which suggests that polar codes based on Arıkan's original $2 \times 2$ kernel might inherently fall short of the optimal scaling exponent of 2.

For larger kernels, effective upper bounds on the scaling exponent are harder to establish as the local evolution of the channels is more complex. In fact, to the best of our knowledge, there is no such explicit bound in the literature, for any kernel of size bigger than $2{ }^{4}$ The analysis of polar codes is a lot more tractable for the case of erasure channels, where symbols get erased (replaced by a '?' but never corrupted). Next we describe some results for erasure channels as well as the difficulty in extending these results to channels such as the BSC.

\subsection{Polar Codes for Erasure Channels}

For the erasure channel, we have analyses of larger kernels and even codes with scaling exponent approaching 2 . Binary $\ell \times \ell$ kernels for powers of two $\ell \leq 64$ optimized for the binary erasure channel appear in [8, 19, 32]; a $64 \times 64$ kernel achieving $\mu<3$ is reported in [32].

Pfister and Urbanke proved in [22] that the optimal scaling exponent $\mu=2$ can be approached if one considers transmission over the $q$-ary erasure channel for large alphabet size $q$. They used polar codes based on $q \times q$ kernels. Fazeli, Hassani, Mondelli, and Vardy [7] then established a similar result for the more challenging and also more interesting case of $q=2$, i.e., for the binary erasure channel, using $\ell \times \ell$ kernels for large $\ell$. They pose proving an analogous result for arbitrary BMS channels as an important challenge. Their conjecture that this can be accomplished provided some of the impetus for our work. Our analysis structure follows a similar blueprint to [7] though the technical ingredients become significantly more complex for channels other than the BEC, as explained next.

The polarization process for erasure channels has a particularly nice structure. If the initial channel $W$ is the binary erasure channel with erasure probability $z$ (denoted $\operatorname{BEC}(z))$, then the Arikan channels $W_{i}, i \in[\ell]$, arising from the linear transformation by the kernel are also binary erasure channels (specifically, $\operatorname{BEC}\left(p_{i}(z)\right)$ where $p_{i}(\cdot)$ are some polynomials of degree at most $\ell$ ). Crucially, all the channels in the recursive tree remain BEC. Therefore it suffices to prove the existence of a good polarizing kernel for the class of binary erasure channels, which is parametrized by a single number, the erasure probability, which also equals the entropy of the channel. As shown in [7], a random kernel works with good

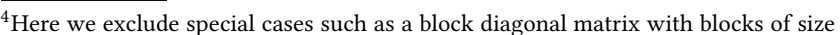
at most 2 which can be reduced to the $2 \times 2$ case but will only have a worse scaling exponent.
} 
probability for all BEC universally. However, fundamentally the calculations for BEC revolve around the rank of various random subspaces, as decoding under the BEC is a linear-algebraic task. Moving beyond the BEC takes us outside the realm of linear algebra into information-theoretic settings where tight quantitative results are much harder to establish.

\subsection{The Road to BSC: Using Multiple Kernels}

For the case when the initial channel $W$ is a BSC, a fundamental difficulty (among others) is that the channels in the recursion tree will no longer remain BSC (even after the first step). Further, to the best of our knowledge, the various channels that arise do not share a nice common exploitable structure. Therefore, we have to think of the intermediate channels as arbitrary BMS channels, a very large and diverse class of channels. It is not clear (to us) if there exists a single kernel to universally polarize all BMS channels at a rapid rate. Even if such a kernel exists, proving so seems out of reach of current techniques. Finally, even for a specific BMS, proving that a random kernel polarizes it fast enough requires some very strong quantitative bounds on the performance and limitations of random linear codes for channel coding. We next describe these issues dealing with which constitutes the core of our contributions.

Since we are not able to establish that a single kernel could work for the whole construction universally, our idea is to pick different kernels, which depend on the channels that we face during the procedure. That way, by picking a suitable kernel for each channel in the tree, we can ensure that polarization is fast enough throughout the whole process.

Though we use different kernels in the code construction, all of them have the same size $\ell \times \ell$. We say that a kernel is good if all but a $\widetilde{O}\left(\ell^{-1 / 2}\right)$ fraction of the bit-channels obtained after polar transform by this kernel have entropy $\ell^{-\Omega(\log \ell)}$-close to either 0 or 1. Given a BMS channel $W$, the code construction consists of $t$ steps, from Step 0 to Step $t-1$. At Step 0, we find a good kernel $K_{1}^{(0)}$ for the original channel $W$. After the polar transform of $W$ using kernel $K_{1}^{(0)}$, we obtain $\ell$ bit-channels $W_{1}, \ldots, W_{\ell}$. Then in Step 1 , we find good kernels for each of these $\ell$ bit-channels. More precisely, the good kernel for $W_{i}$ is denoted as $K_{i}^{(1)}$, and the bitchannels obtained by polar transforms of $W_{i}$ using kernel $K_{i}^{(1)}$ are denoted as $\left\{W_{i, j}: j \in[\ell]\right\}$; see Figure 1 for an illustration. At Step $j$, we will have $\ell^{j}$ bit-channels $\left\{W_{i_{1}, \ldots, i_{j}}: i_{1}, \ldots, i_{j} \in[\ell]\right\}$. For each of them, we find a good kernel $K_{i_{1}, \ldots, i_{j}}^{(j)}$. After polar transform of $\left\{W_{i_{1}, \ldots, i_{j}}: i_{1}, \ldots, i_{j} \in[\ell]\right\}$ using these kernels, we will obtain $\ell^{j+1}$ bit-channels. Finally, after the last step (Step $t-1)$, we will obtain $N=\ell^{t}$ bit-channels $\left\{W_{i_{1}, \ldots, i_{t}}: i_{1}, \ldots, i_{t} \in[\ell]\right\}$. Using the good kernels we obtained in this code construction procedure, we can build an $N \times N$ matrix (or we can view it as a large kernel) $M^{(t)}$ such that the $N$ bit-channels resulting from the polar transform of the original channel $W$ using this large kernel $M^{(t)}$ are exactly $\left\{W_{i_{1}, \ldots, i_{t}}: i_{1}, \ldots, i_{t} \in[\ell]\right\}$. We will say a few more words about this in Section 2.7, and all the details are provided in the full version of this paper.

Define now a random process by $W^{(0)}=W$ and $W^{(j)}=W_{i}^{(j-1)}$ for $i$ uniformly chosen from $[\ell]$, where $W_{i}^{(j-1)}$ is the $i$ th Arrkan bit-channel of $W^{(j-1)}$ with respect to the appropriate kernel chosen in the construction phase. In other words, this is a random process of going down the tree of channels, where a uniformly random child of a current node is taken at each step. Finally, define another random process $H^{(j)}:=H\left(W^{(j)}\right)$. Since every kernel in the construction is chosen to be invertible, $H^{(j)}$ is a martingale due to the conservation of entropy property (2). It is clear that $W^{(j)}$ marginally is a uniformly random channel of the $j^{\text {th }}$ level of channel tree, and then $H^{(j)}$ is the entropy of such a randomly chosen channel.

\subsection{Analysis of Polarization via Recursive Potential Function}

The principle behind polarization is that for large enough $t$, almost all of the channels on the $t$ 's-level of the tree from Figure 1 will be close to either the useless or noiseless channel, i.e., their entropy is very close to 1 or 0 , correspondingly. To estimate how fast such polarization actually happens, one needs to bound the fraction of channels on $t^{\text {th }}$ level that are not yet sufficiently polarized, i.e., $\mathbb{P}\left[H^{(t)} \in(\zeta, 1-\zeta)\right]$ for some tiny threshold $\zeta$, and show that this fraction vanishes rapidly with increasing $t$.

Specifically, we have the following result (stated explicitly in [5, Theorem A.3]) already alluded to in Section 2.1: if for all $t$

$$
\mathbb{P}\left[H^{(t)} \in\left(p \ell^{-t}, 1-p \ell^{-t}\right)\right] \leq D \cdot \beta^{t},
$$

then this corresponds to a polar code with block length $N=\ell^{t}$, rate $\left(D \cdot \beta^{t}+p \ell^{-t}\right)$-close to the capacity of the channel, and decoding error at most $p$ under the successive cancellation decoder.

To track the fractions of polarized and non-polarized channels at each level of the construction, we use a potential function $g_{\alpha}(h)=$ $(h(1-h))^{\alpha}$ for some small fixed $\alpha>0$, which was also used in [20] and [7]. Specifically, we are going to track $\mathbb{E}\left[g_{\alpha}\left(H^{(t)}\right]\right.$ as $t$ increases, since Markov's inequality implies

$$
\begin{array}{r}
\mathbb{P}\left[H^{(t)} \in\left(p \ell^{-t}, 1-p \ell^{-t}\right)\right]=\mathbb{P}\left[g_{\alpha}\left(H^{(t)}\right) \geq g_{\alpha}\left(p \ell^{-t}\right)\right] \\
\leq \frac{\mathbb{E}\left[g_{\alpha}\left(H^{(t)}\right)\right]}{g_{\alpha}\left(p \ell^{-t}\right)} \leq 2\left(\ell^{t} / p\right)^{\alpha} \cdot \mathbb{E}\left[g_{\alpha}\left(H^{(t)}\right)\right] .
\end{array}
$$

To upper bound $\mathbb{E}\left[g_{\alpha}\left(H^{(t)}\right)\right]$ we choose kernels in our construction so that the average of the potential function of all the children of any channel in the tree decreases significantly with respect to the potential function of this channel. Precisely, we want for any channel $W^{\prime}$ in the tree

$$
\underset{i \sim[\ell]}{\mathbb{E}}\left[g_{\alpha}\left(H\left(W_{i}^{\prime}\right)\right)\right] \leq \lambda_{\alpha} \cdot g_{\alpha}\left(H\left(W^{\prime}\right)\right),
$$

where $W_{i}^{\prime}$ are the children of $W^{\prime}$ in the construction tree for $i \in[\ell]$, and the constant $\lambda_{\alpha}$ only depends on $\alpha$ and $\ell$, but is universal for all the channels in the tree (and thus for all the kernels chosen during the construction). If one can guarantee that (5) holds throughout the 

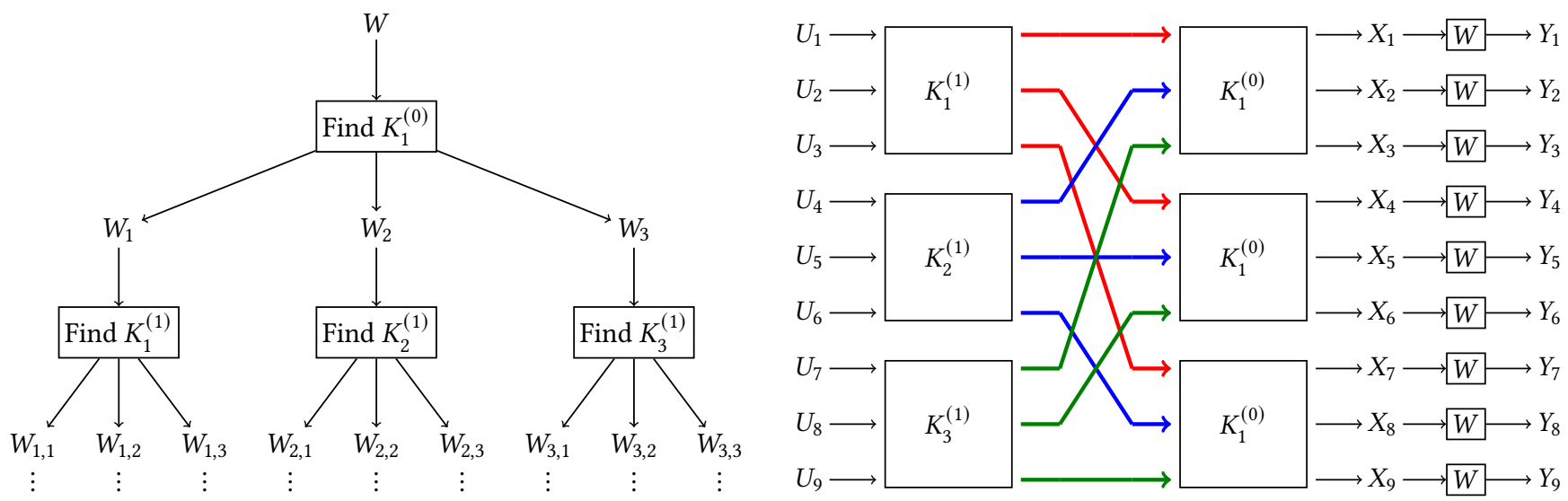

Figure 1: The left figure illustrates the code construction, and the right figure shows the encoding procedure for the special case of $\ell=3$ and $t=2$. All the kernels in this figure have size $3 \times 3$. One can show that the bit-channel $W_{i, j}$ in the left figure is exactly the channel mapping from $U_{3(i-1)+j}$ to $\left(\boldsymbol{U}_{[1: 3(i-1)+j-1]}, \boldsymbol{Y}_{[1: 9]}\right)$ in the right figure.

construction process, then for the martingale process $H^{(t)}$ obtain

$$
\begin{aligned}
\left.\mathbb{E}\left[g_{\alpha}\left(H^{(t)}\right)\right]=\mathbb{E} \underset{j \sim[\ell]}{\mathbb{E}}\left[g_{\alpha}\left(H\left(W_{j}^{(t-1)}\right)\right)\right] \mid W^{(t-1)}\right] \\
=\mathbb{E}\left[\frac{1}{\ell} \frac{\sum_{j=1}^{\ell} g_{\alpha}\left(H\left(W_{j}^{(t-1)}\right)\right)}{g_{\alpha}\left(H\left(W^{(t-1)}\right)\right)} \cdot g_{\alpha}\left(H\left(W^{(t-1)}\right)\right) \mid W^{(t-1)}\right] \\
\leq \lambda_{\alpha} \cdot \mathbb{E}\left[g_{\alpha}\left(H^{(t-1)}\right)\right],
\end{aligned}
$$

and then inductively

$$
\begin{aligned}
\mathbb{E}\left[g_{\alpha}\left(H^{(t)}\right)\right] & \leq \lambda_{\alpha} \cdot \mathbb{E}\left[g_{\alpha}\left(H^{(t-1)}\right)\right] \\
& \leq \lambda_{\alpha}^{2} \cdot \mathbb{E}\left[g_{\alpha}\left(H^{(t-2)}\right)\right] \leq \cdots \leq \lambda_{\alpha}^{t} H^{(0)} \leq \lambda_{\alpha}^{t} .
\end{aligned}
$$

Then (4) and (3) imply existence of code with rate $O\left((N / p)^{\alpha} \cdot \lambda_{\alpha}^{t}\right)$-close to capacity of the channel. Since our main task is to achieve a gap which is close to $N^{-1 / 2}=\ell^{-t / 2}$, we need to argue that it is possible to choose kernels at each step in the construction so that (5) always holds for some $\alpha \rightarrow 0$ and $\lambda_{\alpha} \approx \ell^{-1 / 2}$.

\subsection{Sharp Transition in Polarization}

The main technical contribution of this paper consists in showing that if $\ell$ is large enough, it is possible to choose kernels in the construction process for which $\lambda_{\alpha}$ is close to $\ell^{-1 / 2}$. Specifically, we prove that if $\ell$ is a power of 2 such that $\log \ell=\Omega\left(\frac{1}{\alpha^{1.01}}\right)$, then it is possible to achieve

$$
\lambda_{\alpha} \leq \ell^{-1 / 2+5 \alpha} .
$$

To obtain such a behavior, while choosing the kernel for the current channel $W^{\prime}$ during the recursive process we differentiate between two cases:

Case 1: $W^{\prime}$ is already very noisy or almost noiseless. Such regime is called suction at the ends (following [5]), and it is known that polarization happens (much) faster for this case. So in this case we take a standard Arikan's polarization kernel $K=\left(\begin{array}{ll}1 & 0 \\ 1 & 1\end{array}\right)^{\otimes \log \ell}$ and prove (5) with a geometric decrease factor $\lambda_{\alpha} \leq \ell^{-1 / 2}$.

Case 2: $W^{\prime}$ is neither very noisy nor almost noiseless. We refer to this case as variance in the middle regime (following [5] again). For such a channel we adopt the framework from [7] and show a sharp transition in polarization for a random kernel $K$ and $W^{\prime}$. Specifically, we prove that with high probability over $K \sim\{0,1\}^{\ell \times \ell}$ (for $\ell$ large enough) it holds

$$
\begin{array}{ll}
H\left(W_{i}^{\prime}(K)\right) \leq \ell^{-\Omega(\log \ell)} & \text { for } \quad i \geq \ell \cdot H\left(W^{\prime}\right)+\Omega\left(\ell^{1 / 2} \log ^{3} \ell\right), \\
H\left(W_{i}^{\prime}(K)\right) \geq 1-\ell^{-\Omega(\log \ell)} & \text { for } \quad i \leq \ell \cdot H\left(W^{\prime}\right)-\Omega\left(\ell^{1 / 2} \log ^{3} \ell\right) .
\end{array}
$$

It then follows that only about $\widetilde{O}\left(\ell^{-1 / 2}\right)$ fraction of bit-channels are not polarized for some kernel $K$, which then easily translates into the bound (7) on $\lambda_{\alpha}$ that we desire. Note that we can always ensure that we take an invertible kernel $K$, since a random binary matrix is invertible with at least some constant probability.

Proving such a sharp transition constitutes bulk of the technical work in this paper. In Section 4.2 we show that inequalities in (8) correspond to decoding a single bit of a message which is transmitted through $W^{\prime}$ using a random linear code. The first set of inequalities in (8) then correspond to saying that one can decode this single bit with low error probability with high probability over the randomness of the code, if the rate of the code is at least approximately $\ell^{-1 / 2}$ smaller than the capacity of the channel (where $\ell$ is the blocklength of the code). This follows from the well-studied fact that random linear codes achieve Shannon's capacity over any BMS ([12], [3]).

The second set of inequalities, on the other hand, corresponds to saying that for random linear codes with rate exceeding capacity by at least $\approx \ell^{-1 / 2}$, even predicting a single bit of the message with tiny advantage over a uniform guess is not possible. While it follows from converse Shannon's coding theorem that decoding the entire message is not possible (even with small success probability) for any code above capacity, it does not follow that one cannot recover a particular message bit with accuracy noticeably better 
than random guessing. In fact, if we only want to decode a specific message bit and we do not put any constraints on the code, then we can easily construct codes with rate substantially above capacity that still allow us to decode this specific message bit with high probability. All we need to do here is to repeat the message bit sufficiently many times in the codeword, decode each copy based on the corresponding channel output, and then take a majority vote. The overal code rate does not even figure in this argument. Therefore, one can only hope that the converse theorem for bitdecoding holds for certain code ensembles, and for the purpose of this paper, we restrict ourselves to random linear code ensemble. While the converse for bit-decoding in this case is surely intuitive, establishing it in the strong quantitative form (8) that we need, and also for all BMS channels, turns out to be a challenging task. We present the proof of the strong converse theorem for bit-decoding for the BSC case and describe how to extend it to the general BMS channel case in Section 5.

\subsection{Encoding and Decoding}

Once we have obtained the kernels in the code construction (see Section 2.4), the encoding procedure is pretty standard; see [4, 11, 24, 29, 33] for discussions on polar codes using multiple kernels. As mentioned in Section 2.4, we can build a $N \times N$ ma$\operatorname{trix} M^{(t)}:=D^{(t-1)} Q^{(t-1)} D^{(t-2)} Q^{(t-2)} \ldots D^{(1)} Q^{(1)} D^{(0)}$, where the matrices $Q^{(1)}, Q^{(2)}, \ldots, Q^{(t-1)}$ are some permutation matrices, and $D^{(0)}, D^{(1)}, \ldots, D^{(t-1)}$ are block diagonal matrices. In particular, all the blocks on the diagonal of $D^{(j)}$ are the kernels that we obtained in Step $j$ of the code construction. We illustrate the special case of $\ell=3$ and $t=2$ in Figure 1 . We take a random vector $\boldsymbol{U}_{[1: N]}$ consisting of $N=\ell^{t}$ i.i.d. Bernoulli-1/2 random variables and we transmit the random vector $\boldsymbol{X}_{[1: N]}$ through $N$ independent copies of $W$. Denote the output vector as $\boldsymbol{Y}_{[1: N]}$. Then for every $i \in[N]$, the bit-channel mapping from $U_{i}$ to $\left(\boldsymbol{U}_{[1: i-1]}, \boldsymbol{Y}_{[1: N]}\right)$ is exactly $W_{i_{1}, \ldots, i_{t}}$, where $\left(i_{1}, \ldots, i_{t}\right)$ is $\ell$-ary expansion of $i$.

We have shown that almost all of the $N$ bit-channels $\left\{W_{i_{1}, \ldots, i_{t}}\right.$; $\left.i_{1}, \ldots, i_{t} \in[\ell]\right\}$ become either noiseless or completely noisy. In the code construction, we can track $H\left(W_{i_{1}, \ldots, i_{t}}\right)$ for every $\left(i_{1}, \ldots, i_{t}\right) \in$ $[\ell]^{t}$, and this allows us to identify which $U_{i}$ 's are noiseless under successive decoding. Then in the encoding procedure, we only put information in these noiseless $U_{i}$ 's and set all the other $U_{i}$ 's to be some "frozen" value, e.g., 0 . This is equivalent to saying that the generator matrix of our code is the submatrix of $M^{(t)}$ consisting of rows corresponding to indices $i$ of the noiseless $U_{i}$ 's. It is possible to show that similarly to the classical polar codes, both the encoding and decoding of our code also have $O(N \log N)$ complexity.

As a final remark, we mention that we need to quantize every bit-channel we obtain in every step of the code construction. More precisely, we merge the output symbols whose log-likelihood ratios are close to each other, so that after the quantization, the output alphabet size of every bit-channel is always polynomial in $N$. This allows us to construct the code in polynomial time. Without quantization, the output alphabet size would eventually be exponential in $N$. We will provide more details about this aspect, and how it affects the code construction and the analysis of decoding, in Section 4.1.

\subsection{Proving the Main Theorem}

The main theorem (Theorem 1.1) follows directly from combining the results described in the previous sections. Namely, by using (6) in (4) with $\lambda_{\alpha} \leq \ell^{-1 / 2+5 \alpha}$ from (7), we derive

$$
\mathbb{P}\left[H^{(t)} \in\left(\frac{p}{N}, 1-\frac{p}{N}\right)\right] \leq \frac{2 N^{-1 / 2+6 \alpha}}{p^{\alpha}} .
$$

By taking $p$ inverse polynomial in $N, p=O\left(\frac{1}{N^{c}}\right)$, and using the standard argument from [5, Theorem A.3], we obtain the polar code with rate being $O\left(N^{-1 / 2+(c+6) \alpha}\right)$-close to capacity and inversepolynomial decoding error probability $p$. Section 2.7 shows that encoding and decoding can be done in $O(N \log N)$ time, and it can be also shown that the construction is done in $\operatorname{poly}(N)$ time.

As it is mentioned at the end of previous section, we use approximations of the bit-channels during the construction phase, to keep the construction poly-time. This leads to only being able to obtain inverse-polynomial decoding error probability using this approach (since the parameters of the approximated bit-channels are only inverse-polynomially close approximations to the parameters of the true bit-channels). However, it is possible to use the fact that we take Arikan's polarization kernels for the suction at the end regime (see Case 1 in Section 2.6) to argue that the parameters (entropy of Bhattacharrya parameter) of some true bit-channel is extremely small, even only if we tracked the approximation of this bit-channel, which can only have inverse-polynomially small parameters. Using this idea and a tight analysis from [30], we can drive down the decoding error probability to be exponentially small in $N$, while still keeping the construction time polynomial in $N$. More details and full proofs can be found in the full version of this paper.

\section{PRELIMINARIES}

\subsection{Binary Entropy Function}

All the logarithms in this paper are to the base 2 . The binary entropy function is defined as $h(x)=x \log \frac{1}{x}+(1-x) \log \frac{1}{1-x}$ for $x \in[0,1]$, where $0 \log 0$ is taken to be 0 . We will use a simple fact that $h(x) \leq$ $2 x \log \frac{1}{x}$ for $x \in[0,1 / 2)$ several times in the proofs. The following proposition follows from the facts that $h(x)$ is concave, increasing for $x \in[0,1 / 2)$, and symmetric around $1 / 2$, i.e. $h(x)=h(1-x)$ for $x \in[0,1]$.

Proposition 3.1. For any $x, y \in[0,1],|h(x)-h(y)| \leq h(|x-y|)$.

\subsection{Channel Degradation}

Definition 3.2. Let $W:\{0,1\} \rightarrow y$ and $\widetilde{W}:\{0,1\} \rightarrow \widetilde{y}$ be two BMS channels. We say that $\widetilde{W}$ is degraded with respect to $W$, or, correspondingly, $W$ is upgraded with respect to $\widetilde{W}$, denoted as $\widetilde{W} \leq W$, if there exists a discrete memoryless channel $W_{1}: \mathcal{Y} \rightarrow \widetilde{\mathcal{Y}}$ such that

$$
\widetilde{W}(\widetilde{y} \mid x)=\sum_{y \in \mathcal{Y}} W(y \mid x) W_{1}(\widetilde{y} \mid y) \quad \forall x \in\{0,1\}, \widetilde{y} \in \widetilde{\mathcal{y}} .
$$

Note that this is equivalent to saying that $\widetilde{W}(x)$ and $W_{1}(W(x))$ are identically distributed for any $x \in\{0,1\}$. In other words, one can simulate the usage of $\widetilde{W}$ by first using the channel $W$ and then applying some other channel $W_{1}$ to the output of $W$ to get a final 


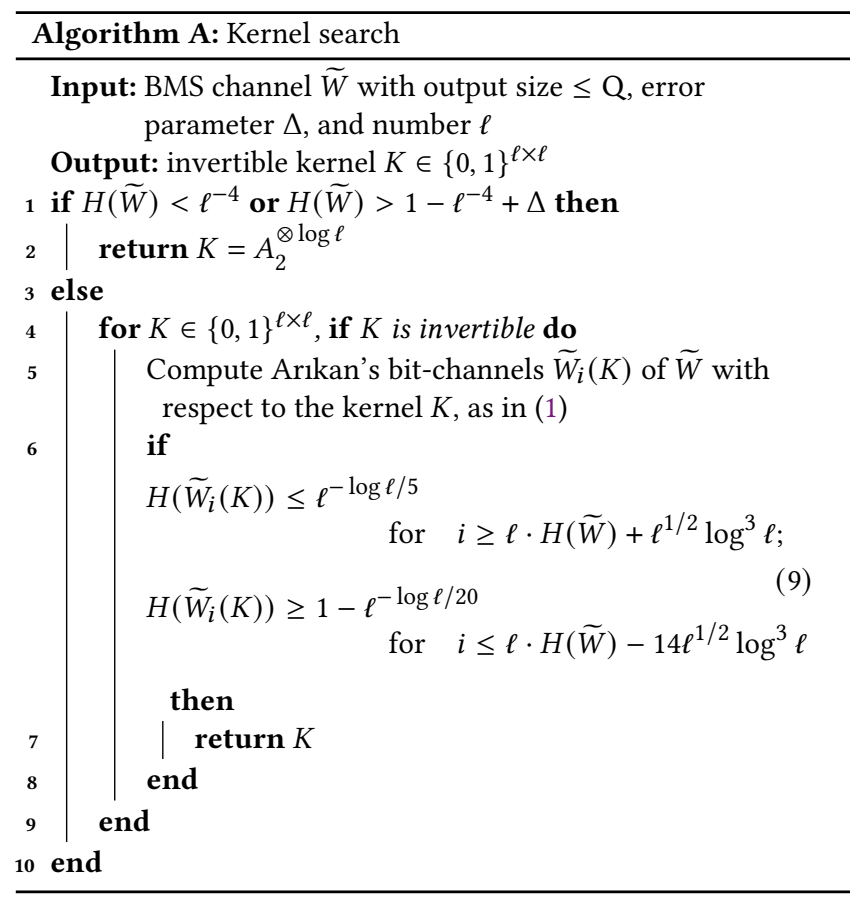

output. We will use some useful facts from [28, Lemma 3] and [33, Lemma IV.1]

Proposition 3.3. Let $W$ and $\widetilde{W}$ be two BMS channels, such that $\widetilde{W} \leq W$. Then $H(\widetilde{W}) \geq H(W)$.

Proposition 3.4. Let $W$ and $\widetilde{W}$ be BMS channels, such that $\widetilde{W} \leq W$, and $K \in\{0,1\}^{\ell \times \ell}$ be any invertible matrix. Denote by $W_{i}, \widetilde{W}_{i}$ the Arlkan's bit-channels of $W$ and $\widetilde{W}$ with respect to the kernel $K$ for any $i \in[\ell]$. Then for any $i \in[\ell]$, we have $\widetilde{W}_{i} \leq W_{i}$, and consequently $H\left(\widetilde{W}_{i}\right) \geq H\left(W_{i}\right)$.

\section{GIVE ME A CHANNEL, I'LL GIVE YOU A KERNEL}

In this section we show that for any given binary-input memoryless symmetric (BMS) channel $W$ we can find a kernel $K$ of size $\ell \times \ell$, such that the Arıkan's bit-channels of $W$ with respect to this kernel will be highly polarized. By this we mean that the multiplicative decrease $\lambda_{\alpha}$ defined in (5) will be sufficiently close to $\ell^{-1 / 2}$. The algorithm (Algorithm $A$ ) to find such a kernel is as follows: if the channel is already almost noiseless or too noisy (entropy is very close to 0 or 1 ), we take this kernel to be a tensor power of original Arıkan's kernel for polar codes, $A_{2}=\left(\begin{array}{ll}1 & 0 \\ 1 & 1\end{array}\right)$. Otherwise, the algorithm will just try out all the possible invertible kernels in $\{0,1\}^{\ell \times \ell}$, until a "good" kernel is found, which means that conditions (9) should be satisfied. Before proving that Algorithm A achieves our goals of bringing $\lambda_{\alpha}$ close to $\ell^{-1 / 2}$, we discuss several details about it.

\subsection{Local Kernel Construction}

As briefly discussed at the end of Section 2.7, we are unable to efficiently track all the bit-channels in the $\ell$-ary recursive tree exactly.
This is because the size of the output alphabet of the channels increase exponentially after each step deeper into the tree (this simply follows from the definition of bit-channels (1)). Thus computing all the channels (and their entropies) cannot be done in $\operatorname{poly}(N)$ time. To overcome this issue we follow the approach of [28], with subsequent simplification in [14], of approximating the channels in the tree by degrading them. Degradation is achieved via the procedure of merging the output symbols, which (a) decreases the output alphabet size, and (b) does not change the entropy of the channel too much. This implies (with all the details worked out in full version of the paper) that we can substitute all the channels in the tree of depth $t$ by their degraded approximations, such that all the channels has output alphabet size at most $\mathrm{Q}$ (a parameter depending on $N=\ell^{t}$ to be chosen), and that if $\widetilde{W}$ is a degraded approximation of the channel $W$ in the tree, than $H(W) \leq H(\widetilde{W}) \leq H(W)+\Delta$ for some $\Delta$ depending on $\mathrm{Q}$. Moreover, in Theorem 4.1 which we formulate shortly, we show that when we apply the Algorithm A to a degraded approximation $\widetilde{W}$ of $W$ with small enough $\Delta$, then, even though conditions (9) only dictate a sharp transition for $\widetilde{W}$, the same kernel will induce a sharp transition in polarization for $W$.

The second issue which such degraded approximation resolves is the running time of the Algorithm A. Notice that we are only going to apply it for channels with output size bounded by $\mathrm{Q}$, and recall that we think of $\ell$ as of a constant (though very large). First of all, trying out all the possible kernels will then also take a constant number of iterations. Finally, within each iteration, just calculating all the Arıkan's bit-channels and their entropies in a straightforward way will take poly $\left(Q^{\ell}\right)$ time, which is just poly $(Q)$ when we treat $\ell$ as a constant. Therefore by choosing Q to be polynomial in $N$, the algorithm indeed works in $\operatorname{poly}(N)$ time.

We now proceed with showing that the Algorithm A always returns a kernel which makes $\lambda_{\alpha}$ from (5) close to $\ell^{-1 / 2}$.

Theorem 4.1. Let $\alpha>0$ be a small constants. Let $\ell$ be a power of 2 such that $\log \ell \geq \frac{11}{\alpha}$ and $\frac{\log \ell}{\log \log \ell+2} \geq \frac{3}{\alpha}$. Let $W:\{0,1\} \rightarrow y$ and $\widetilde{W}:\{0,1\} \rightarrow \widetilde{\mathcal{Y}}$ be two BMS channels, such that $\widetilde{W} \leq W$, $H(\widetilde{W})-\Delta \leq H(W) \leq H(\widetilde{W})$ for some $0 \leq \Delta \leq \ell^{-\log \ell}$, and $|\widetilde{\mathcal{Y}}| \leq$ $\mathrm{Q}$. Then the Algorithm $A$ on input $\widetilde{W}, \Delta$, and $\ell$ returns a kernel $K \in\{0,1\}^{\ell \times \ell}$ that satisfies

$$
\frac{1}{\ell \cdot g_{\alpha}(H(W))} \sum_{i=1}^{\ell} g_{\alpha}\left(H\left(W_{i}\right)\right) \leq \ell^{-\frac{1}{2}+5 \alpha},
$$

where $W_{1}, W_{2}, \ldots, W_{\ell}$ are the Arlkan's bit-channels of $W$ with respect to the kernel $K$, and the function $g_{\alpha}(\cdot)$ is defined as $g_{\alpha}(h)=$ $(h(1-h))^{\alpha}$ for any $h \in[0,1]$.

Proof. As we discussed above, we consider two cases:

Suction at the ends. If $H(\widetilde{W}) \notin\left(\ell^{-4}, 1-\ell^{-4}+\Delta\right)$, the Algorithm A returns a standard Arıkan's kernel $K=A_{2}^{\otimes \log \ell}$ on input $\widetilde{W}$ and $\Delta$. For this case $H(W) \notin\left(\ell^{-4}, 1-\ell^{-4}\right)$, and fairly standard arguments imply that the polarization under such a kernel is much faster when the entropy is close to 0 or 1.The full proofs for this case can be found in the full version of the paper. 
Variance in the middle. Otherwise, if $H(\tilde{W}) \in\left(\ell^{-4}, 1-\right.$ $\left.\ell^{-4}+\Delta\right)$, it holds $H(W) \in\left(\ell^{-4}-\Delta, 1-\ell^{-4}+\Delta\right)$, thus $H(W) \in$ $\left(\ell^{-4} / 2,1-\ell^{-4} / 2\right)$.

We first need to argue that the algorithm will at least return some kernel. This argument is one of the main technical contributions of this work, and we formulate it as Theorem 4.3 in Section 4.2. The theorem essentially claims that for any $\widetilde{W}$ an overwhelming fraction of possible kernels $K \in\{0,1\}^{\ell \times \ell}$ satisfies the conditions in (9) for $\widetilde{W}$ and $K$ (note that we do not use any conditions on the size of $\widetilde{\mathcal{y}}$ or the entropy $H(\widetilde{W})$ at all at this point). Clearly then, there is a decent fraction of invertible kernels from $\{0,1\}^{\ell \times \ell}$ which also satisfy these conditions. Therefore, the algorithm will indeed terminate and return such a good kernel. Moreover, since the theorem claims that a random kernel from $\{0,1\}^{\ell \times \ell}$ will satisfy (9) with high probability, and it is also known that it will be invertible with at least some constant probability. It means that instead of iterating through all possible kernels in step 4 of the Algorithm A, we could take a random kernel and check it, and then the number of iterations needed to find a good kernel would be very small with high probability. However, to keep everything deterministic, we stick to the current approach.

Suppose now the algorithm returned an invertible kernel $K \in$ $\{0,1\}^{\ell \times \ell}$, which means that relations (9) hold for $\widetilde{W}$ and Arıkan's bit-channels $\widetilde{W}_{1}, \widetilde{W}_{2}, \ldots, \widetilde{W}_{\ell}$ (we omit dependence on $K$ from now on). Denote also $W_{i}=W_{i}(K)$ as an Arıkan's bit-channels of $W$ with respect to $K$. First, since degradation is preserved after considering Arrkan's bit-channels according to Proposition3.4, $\widetilde{W}_{i} \leq W_{i}$, thus $H\left(W_{i}\right) \leq H\left(\widetilde{W}_{i}\right)$ for all $i \in[\ell]$. Now, since $K$ is invertible, conservation of entropy implies $\sum_{i=1}^{\ell}\left(H\left(\widetilde{W}_{i}\right)-H\left(W_{i}\right)\right)=$ $\ell(H(\widetilde{W})-H(W)) \leq \ell \cdot \Delta$, therefore derive $H\left(W_{i}\right) \leq H\left(\widetilde{W}_{i}\right) \leq$ $H\left(W_{i}\right)+\ell \cdot \Delta$ for any $i \in[\ell]$. Then we deduce

$$
\begin{aligned}
& H\left(W_{i}\right) \leq H\left(\widetilde{W}_{i}\right) \leq \ell^{-\log \ell / 5} \\
& \quad \text { for } \quad i \geq \ell \cdot H(\widetilde{W})+\ell^{1 / 2} \log ^{3} \ell ; \\
& H\left(W_{i}\right) \geq H\left(\widetilde{W}_{i}\right)-\ell \cdot \Delta \geq 1-\ell^{-\log \ell / 21} \\
& \quad \text { for } i \leq \ell \cdot H(\widetilde{W})-14 \ell^{1 / 2} \log ^{3} \ell,
\end{aligned}
$$

where we used that we chose $\Delta \leq \ell^{-\log \ell}$ in the condition of the theorem.

Recall that $H(W) \in\left(\ell^{-4} / 2,1-\ell^{-4} / 2\right)$ for variance in the middle regime, and note that this implies $g_{\alpha}(H(W)) \geq g_{\alpha}\left(\ell^{-4} / 2\right) \geq \frac{1}{2} \ell^{-4 \alpha}$. Using (11) and the trivial bound $g_{\alpha}(x) \leq 1$ we obtain that the LHS of the desired inequality (10) is at most

$$
\begin{aligned}
\frac{1}{\ell \cdot g_{\alpha}(H(W))}( & \sum_{i=1}^{\ell \cdot H(\widetilde{W})-14 \cdot \ell^{1 / 2} \log ^{3} \ell} g_{\alpha}\left(1-\ell^{-\log \ell / 21}\right) \\
& \left.+15 \ell^{1 / 2} \log ^{3} \ell+\sum_{i=\ell \cdot H(\widetilde{W})+\ell^{1 / 2} \log ^{3} \ell}^{\ell} g_{\alpha}\left(\ell^{-\log \ell / 5}\right)\right) \\
& <30 \ell^{-\frac{1}{2}+4 \alpha} \log ^{3} \ell+2 \ell^{-\alpha \log \ell / 21+4 \alpha}<\ell^{-\frac{1}{2}+5 \alpha},
\end{aligned}
$$

where the last inequality uses the conditions $\log \ell \geq \frac{11}{\alpha}$ and $\frac{\log \ell}{\log \log \ell+2} \geq \frac{3}{\alpha}$ that we have on $\ell$.
Remark 4.2. We are interested in the cases where $\alpha$ is very close to 0 . For such $\alpha$, we can absorb the two conditions on $\ell$ in Theorem 4.1 into one condition $\log \ell \geq \alpha^{-1.01}$.

\subsection{Strong Channel Coding and Converse Theorems}

In this section we will show that Algorithm A, which is used to prove the multiplicative decrease of almost $\ell^{-1 / 2}$ as in (10) in the settings of Theorem 4.1, indeed always returns some kernel for the regime when the entropy of the channel is not close to 0 or 1 . While the analysis of suction at the ends regime is pretty standard and relies on the fact that polarization is getting much faster when the channel is noiseless or useless, in this section we will follow the ideas from [7] and prove a sharp transition in the polarization behaviour, when the polarization happens under a random and sufficiently large kernel.

The sharp transition stems from the fact that when the kernel $K$ is large enough, with high probability (over randomness of $K$ ) all the Arrkan's bit-channel with respect to $K$, except for approximately $\ell^{1 / 2}$ of them in the middle, are guaranteed to be either very noisy or almost noiseless. We formulate the main result of this section in the following theorem, which was used in the proof of Theorem 4.1:

THeOREM 4.3. Let $W$ be any BMS channel, and let $W_{1}, W_{2}, \ldots, W_{\ell}$ be the Arlkan's bit-channels defined in (1) with respect to the kernel $K$ chosen uniformly at random from $\{0,1\}^{\ell \times \ell}$. Then for the following inequalities all hold with probability $\left(1-o_{\ell}(1)\right)$ over the choice of $K$ :

(a) $H\left(W_{i}\right) \leq \ell^{-(\log \ell) / 5} \quad$ for $i \geq \ell \cdot H(W)+\ell^{1 / 2} \log ^{3} \ell$;

(b) $H\left(W_{i}\right) \geq 1-\ell^{-(\log \ell) / 20}$ for $i \leq \ell \cdot H(W)-14 \cdot \ell^{1 / 2} \log ^{3} \ell$.

Remark 4.4. One can notice that the above theorem is stated for any BMS channel $W$, independent of the value of $H(W)$.

The proof of this theorem relies on results concerning bitdecoding for random linear codes that are interesting beyond the connection to polar codes. The following proposition shows how to connect Arıkan's bit-channels to this context.

Proposition 4.5. Let $W$ be a BMS channel, $K \in\{0,1\}^{\ell \times \ell}$ be an invertible matrix, and $i \in[\ell]$. Set $k=\ell-i+1$, and let $G$ be $a$ matrix which is formed by the last $k$ rows of $K$. Let $\boldsymbol{U}$ be a random vector uniformly distributed over $\{0,1\}^{\ell}$, and $\boldsymbol{V}$ be a random vector uniformly distributed over $\{0,1\}^{k}$. Then

$$
H\left(U_{i} \mid W^{\ell}(\boldsymbol{U} \cdot K), \boldsymbol{U}_{<i}\right)=H\left(V_{1} \mid W^{\ell}(\boldsymbol{V} \cdot G)\right)
$$

The proof of this proposition only uses basic properties of BMS channels and linear codes, and can be found in the final version of the paper. Notice now that the LHS of (12) is exactly the entropy $H\left(W_{i}\right)$ of the $i$ 's Arikan's bit-channel of $W$ with respect to the kernel $K$, by definition of this bit-channel. On the other hand, one can think of the RHS of (12) in the following way: look at $G$ as a generator matrix for a linear code of blocklength $\ell$ and dimension $k$, which is transmitted through the channel $W$. Then $H\left(V_{1} \mid W^{\ell}(\boldsymbol{V} \cdot G)\right)$ in some sense corresponds to how well one can decode the first bit of the message, given the output of the channel. Since in Theorem 4.3 
we are interested in random kernels, the generator matrix $G$ is also random, and thus we are indeed interested in understanding bit-decoding of random linear codes.

4.2.1 The BEC Case. When $W$ is the binary erasure channel, a statement very similar to Theorem 4.3 was established in [7]. The situation for the BEC is simpler and we now describe the intuition behind this.

Suppose we map uniformly random bits $\boldsymbol{U} \in\{0,1\}^{\ell}$ to $\boldsymbol{X}=\boldsymbol{U} K$ for a random $\ell \times \ell$ binary matrix $K$. We will observe $\approx(1-z) \ell$ bits of $\boldsymbol{X}$ after it passes through $\operatorname{BEC}(z)$; call these bits $\boldsymbol{Z}$. For a random $K$, with high probability the first $\approx z \ell$ bits of $\boldsymbol{U}$ will be linearly independent of these observed bits $\boldsymbol{Z}$. When this happens we will have $H\left(W_{i}\right)=1$ for $i \lesssim z \ell$. On the other hand, $\boldsymbol{Z}$ together with the first $\approx z \ell$ bits of $\boldsymbol{U}$ will have full rank w.h.p. over the choice of $K$. When this is the case, the remaining bits $U_{i}$ for $i \gtrsim z \ell$ will be determined as linear combinations of these bits, making the corresponding conditional entropies $H\left(W_{i}\right)=0$. Thus except for a few exceptional indices around $i \approx z \ell$, the entropy $H\left(W_{i}\right)$ will be really close to 0 or 1 . The formal details and quantitative aspects are non-trivial as the argument has to handle the case when $z$ is itself close to 0 or 1 , and one has to show the number of exceptional indices to be $\lesssim \sqrt{\ell}$ (which is the optimal bound). But ultimately the proof amounts to understanding the ranks of various random subspaces. When $W$ is a BMS channel, the analysis is no longer linear-algebraic, and becomes more intricate. This is the subject of the rest of this section as well as Section 5.

4.2.2 Part (a): Channel Capacity Theorem. Part (a) of Theorem 4.3 corresponds to transmitting through $W$ random linear codes with rates below the capacity of the channel. For this regime, it turns out that we can use the classical result that random linear codes achieve the capacity of the channel with low error decoding probability. Trivially, the bit-decoding error probability is even smaller, making the corresponding conditional entropy also very small. Therefore, the following theorem follows from classical Shannon's theory:

THEOREM 4.6. Let $W$ be any BMS channel and $k \leq \ell(1-H(W))-$ $\ell^{1 / 2} \log ^{3} \ell$. Let $G$ be a random binary matrix uniform over $\{0,1\}^{k \times \ell}$. Suppose a codeword $\boldsymbol{V} \cdot G$ is transmitted through $\ell$ copies of the channel $W$, where $\boldsymbol{V}$ is uniformly random over $\{0,1\}^{k}$, and let $\boldsymbol{Y}$ be the output vector, i.e. $\mathbf{Y}=W^{\ell}(\boldsymbol{V} \cdot G)$. Then with high probability over the choice of $G$ it holds $H\left(V_{1} \mid \boldsymbol{Y}\right) \leq \ell^{-(\log \ell) / 5}$.

Proof. The described communication is just a transmission of a random linear code $C=\left\{\boldsymbol{v} G, \mathbf{v} \in\{0,1\}^{k}\right\}$ through $W^{\ell}$, where the rate of the code is $R=\frac{k}{\ell} \leq I(W)-\ell^{-1 / 2} \log ^{3} \ell$, so it is separated from the capacity of the channel. It is a wellstudied fact that random (linear) codes achieve capacity for BMS, and moreover a tight error exponent was described by Gallager in [12] and analyzed further in [3], [10], [6]. Specifically, one can show $\overline{P_{e}} \leq \exp \left(-\ell E_{r}(R, W)\right)$, where $\overline{P_{e}}$ is the probability of decoding error, averaged over the ensemble of all linear codes of rate $R$, and $E_{r}(R, W)$ is the so-called random coding exponent. It is proven in [16, Theorem 2.3] that for any BMS channel $W$, one has $E_{r}(R, W) \geq E_{r}^{\mathrm{BSC}}(R, I(W))$ where the latter is the error exponent for the BSC channel with the same capacity $I(W)$ as $W$. But the random scaling exponent for BSC channels for the regime when the rate is close to the capacity of the channel is given by the so-called sphere-packing exponent $E_{r}^{\mathrm{BSC}}(R, I)=E_{\mathrm{sp}}(R, I)$ which is easily shown to be "almost" quadratic in $(I-R)$. Specifically, one can show $E_{\mathrm{sp}}(R, I) \geq \frac{\log ^{4} \ell}{2 \ell}$ when $R \leq I-\ell^{-1 / 2} \log ^{3} \ell$, and therefore $\overline{P_{e}} \leq \exp \left(-\ell E_{r}(R, W)\right) \leq \exp \left(-\ell E_{\mathrm{sp}}(R, I(W))\right) \leq \exp \left(-\log ^{4} \ell / 2\right)$. Then Markov's inequality implies that if we take a random linear code (i.e. choose a random binary matrix $G$ ), then with probability at least $1-\ell^{-2}$ the decoding error is going to be at most $\ell^{2} \exp \left(-\log ^{4} \ell / 2\right) \leq \exp \left(-\log ^{4} \ell / 4\right) \leq \ell^{-\log \ell / 4}$. Consider such a good linear code (matrix $G$ ), and then $\boldsymbol{V}$ can be decoded from $\boldsymbol{Y}$ with high probability, thus, clearly, $V_{1}$ can be recovered from $\boldsymbol{Y}$ with at least the same probability. Then Fano's inequality gives us:

$$
H\left(V_{1} \mid \mathbf{Y}\right) \leq h_{2}\left(\ell^{-\log \ell / 4}\right) \leq \ell^{-\log \ell / 5} .
$$

Thus we indeed obtain that the above holds with high probability (at least $1-\ell^{-2}$, though this is very loose) over the random choice of $G$.

4.2.3 Part (b): Strong Converse for Bit-Decoding under Noisy Channel Coding. On the other hand, part (b) of Theorem 4.3 concerns bit-decoding of linear codes with rates above the capacity of the channel. We prove that with high probability, for a random linear code with rate slightly above capacity of a BMS channel, any single bit of the input message is highly unpredictable based on the outputs of the channel on the transmitted codeword. Formally, we have the following theorem.

THeOREM 4.7. Let $W$ be any BMS channel, $\ell$ and $k$ be any integers that satisfy $\ell \geq k \geq \ell(1-H(W))+14 \ell^{1 / 2} \log ^{3} \ell$. Let $G$ be a random binary matrix uniform over $\{0,1\}^{k \times \ell}$. Suppose a message $\boldsymbol{V} \cdot G$ is transmitted through $\ell$ copies of the channel $W$, where $\boldsymbol{V}$ is uniformly random over $\{0,1\}^{k}$, and let $\boldsymbol{Y}$ be the output vector, i.e. $\boldsymbol{Y}=W^{\ell}(\boldsymbol{V}$. $G)$. Then, with probability at least $1-\ell^{-\log \ell / 20}$ over the choice of $G$ it holds $H\left(V_{1} \mid \mathbf{Y}\right) \geq 1-\ell^{-\log \ell / 20}$.

Since the theorem is of independent interest and of a fundamental nature, we devote a separate Section 5 to present a proof for it.

The above statements make the proof of Theorem 4.3 immediate:

Proof of Theorem 4.3. Denote $k=\ell-i+1$, then by Proposition $4.5 H\left(W_{i}\right)=H\left(V_{1} \mid W^{\ell}\left(\boldsymbol{V} \cdot G_{k}\right)\right)$, where $\boldsymbol{V} \sim\{0,1\}^{k}$ and $G_{k}$ is formed by the last $k$ rows of $K$. Note that since $K$ is uniform over $\{0,1\}^{\ell \times \ell}$, this makes $G_{k}$ uniform over $\{0,1\}^{k \times \ell}$ for any $k$. Then:

(a) For any $i \geq \ell \cdot H(W)+\ell^{1 / 2} \log ^{3} \ell$, we have $k \leq \ell(1-H(W))-$ $\ell^{1 / 2} \log ^{3} \ell$, and therefore Theorem 4.6 applies, giving $H\left(W_{i}\right) \leq$ $\ell^{-(\log \ell) / 5}$ with probability at least $1-\ell^{-2}$ over $K$.

(b) Analogically, if $i \leq \ell \cdot H(W)-14 \cdot \ell^{1 / 2} \log ^{3} \ell$, then $k \geq \ell(1-$ $H(W))+14 \ell^{1 / 2} \log ^{3} \ell$, and Theorem 4.7 gives $H\left(W_{i}\right) \geq 1-$ $\ell^{-(\log \ell) / 20}$ with probability at least $1-\ell^{-(\log \ell / 20)}$ over $K$.

It only remains to take the union bound over all indexes $i$ as in (a) and (b), which implies that all of the bounds on the entropies will hold simultaneously with probability at least $1-\ell \cdot \ell^{-2} \geq 1-\ell^{-1}$ over the random kernel $K$. 


\section{STRONG CONVERSE FOR BSC}

In this section we present a proof of the strong converse theorem for bit-decoding random linear codes (Theorem 4.7) for the case when the underlying channel is the binary symmetric channel (BSC). In particular, we need to show the sharp transition as in (8), when the channel is BSC. The proof for the general BMS channel case follows the same blueprint by using the fact that a BMS channel can be represented as a convex combination of BSC subchannels, but executing it involves overcoming several additional technical hurdles. The proof for arbitrary BMS channel is presented in the full version of this paper.

Let us fist formulate the precise theorem for the binary symmetric channel.

THEOREM 5.1. Let $W$ be the $B S C_{p}$ channel, and let $\ell, k$ be integers that satisfy $\ell \geq k \geq \ell(1-H(W))+\Omega\left(\ell^{1 / 2} \log \ell\right)$. Let $G$ be a random binary matrix uniform over $\{0,1\}^{k \times \ell}$. Suppose a message $\boldsymbol{V} \cdot G$ is transmitted through $\ell$ copies of the channel $W$, where $\boldsymbol{V}$ is uniformly random over $\{0,1\}^{k}$, and let $\boldsymbol{Y}$ be the output vector, i.e. $\boldsymbol{Y}=W^{\ell}(\boldsymbol{V}$. $G)$. Then, with probability at least $1-\ell^{-\Omega(\log \ell)}$ over the choice of $G$ it holds $H\left(V_{1} \mid \mathbf{Y}\right) \geq 1-\ell^{-\Omega(\log \ell)}$.

We want to point out two quantitative features of the above theorem. First, it applies at rates $\approx \Omega\left(\ell^{-1 / 2}\right)$ above capacity. Second, it rules out predicting the bit $V_{1}$ with advantage $\ell^{-\omega(1)}$ over random guessing. Both these features are important to guarantee the desired bound $\lambda_{\alpha} \lesssim \ell^{-1 / 2}$.

Throughout this section consider the channel $W$ to be BSC with the crossover probability $p \leq \frac{1}{2}$. Denote $H=H(W)=h(p)$, where $h(\cdot)$ is the binary entropy function.

Proof of Theorem 5.1. We prove the lower bound on $H\left(V_{1} \mid \boldsymbol{Y}\right)$ by lower bounding $\underset{g \sim G}{\mathbb{E}}\left[H\left(V_{1} \mid \boldsymbol{Y}\right)\right]$ and using Markov's inequality. Thus we write

$$
\begin{aligned}
\underset{g \sim G}{\mathbb{E}}\left[H^{(g)}\left(V_{1} \mid \boldsymbol{Y}\right)\right]=\sum_{g} \mathbb{P}(G=g) H^{(g)}\left(V_{1} \mid \boldsymbol{Y}\right) \\
\quad=\sum_{g} \mathbb{P}(G=g)\left(\sum_{\mathbf{y} \in \mathcal{Y}^{\ell}} \mathbb{P}^{(g)}(\boldsymbol{Y}=\boldsymbol{y}) H^{(g)}\left(V_{1} \mid \boldsymbol{Y}=\boldsymbol{y}\right)\right),
\end{aligned}
$$

where the summation of $g$ is over binary matrices $\{0,1\}^{k \times \ell}$, and by $\mathbb{P}^{(g)}(\cdot)$ and $H^{(g)}(\cdot)$ we denote probability and entropy over the randomness of the message $\boldsymbol{V}$ and channel noise for a fixed matrix g.

Restrict to zero-input. We rewrite

$$
\begin{aligned}
\underset{g \sim G}{\mathbb{E}}\left[H^{(g)}\left(V_{1} \mid \boldsymbol{Y}\right)\right] \\
=\sum_{g} \mathbb{P}(G=g)\left(\sum_{\boldsymbol{y} \in \mathcal{Y}^{\ell}} \mathbb{P}^{(g)}(\boldsymbol{Y}=\boldsymbol{y}) H^{(g)}\left(V_{1} \mid \boldsymbol{Y}=\boldsymbol{y}\right)\right) \\
=\sum_{\mathbf{y} \in \mathcal{Y}^{\ell}} \sum_{g} \mathbb{P}^{(g)}(\boldsymbol{Y}=\boldsymbol{y}) \cdot \mathbb{P}(G=g) H^{(g)}\left(V_{1} \mid \boldsymbol{Y}=\boldsymbol{y}\right) .
\end{aligned}
$$

Our first step is to prove that in the above summation we can change $\mathbb{P}^{(g)}(\boldsymbol{Y}=\boldsymbol{y})$ to $\mathbb{P}^{(g)}(\boldsymbol{Y}=\boldsymbol{y} \mid \boldsymbol{V}=\mathbf{0})$, where $\mathbf{0}$ is the all-zero vector. This observation is crucial for our arguments, since it allows us to only consider the outputs $\boldsymbol{y}$ which are "typical" for the all-zero codeword when approximating $\underset{g \sim G}{\mathbb{E}}\left[H^{(g)}\left(V_{1} \mid \boldsymbol{Y}\right)\right]$. Precisely, we prove

Lemma 5.2. Let $W$ be a BMS channel, $\ell$ and $k$ be integers such that $k \leq \ell$. Let $G$ be a random binary matrix uniform over $\{0,1\}^{k \times \ell}$. Suppose a message $\boldsymbol{V} \cdot G$ is transmitted through $\ell$ copies of $W$, where $\boldsymbol{V}$ is uniformly random over $\{0,1\}^{k}$, and let $\boldsymbol{Y}$ be the output vector $\boldsymbol{Y}=W^{\ell}(\boldsymbol{V} \cdot G)$. Then

$$
\begin{aligned}
\underset{g \sim G}{\mathbb{E}}\left[H^{(g)}\left(V_{1} \mid \boldsymbol{Y}\right)\right] \\
=\sum_{\boldsymbol{y} \in \mathcal{Y}^{\ell}} \sum_{g} \mathbb{P}^{(g)}(\boldsymbol{Y}=\boldsymbol{y} \mid \boldsymbol{V}=\mathbf{0}) \cdot \mathbb{P}(G=g) H^{(g)}\left(V_{1} \mid \boldsymbol{Y}=\boldsymbol{y}\right) .
\end{aligned}
$$

The above lemma is formulated for any BMS channel, and it is also used for the proof of the general case of Theorem 4.7. The proof of this lemma uses the symmetry of linear codes with respect to shifting by a codeword and additive structure of BSC, together with the fact that any BMS channel can be represented as a convex combination of several BSC subchannels.

Notice that $\mathbb{P}^{(g)}(\boldsymbol{Y}=\boldsymbol{y} \mid \boldsymbol{V}=\mathbf{0})$ does not in fact depend on the matrix $g$, since $\mathbf{0} \cdot g=\mathbf{0}$, and so randomness here only comes from the noise which the channel $W$ introduces. Specifically, $\mathbb{P}^{(g)}(\boldsymbol{Y}=$ $\boldsymbol{y} \mid \boldsymbol{V}=\mathbf{0})=p^{w t(y)}(1-p)^{\ell-w t(y)}$, where we denote by $w t(\mathbf{y})$ the Hamming weight of $\boldsymbol{y}$. Then in (13) we obtain

$$
\begin{aligned}
\underset{g \sim G}{\mathbb{E}}\left[H^{(g)}\left(V_{1} \mid \boldsymbol{Y}\right)\right] \\
=\sum_{\boldsymbol{y} \in \mathcal{Y}^{\ell}} p^{w t(\mathbf{y})}(1-p)^{\ell-w t(y)} \underset{g \sim G}{\mathbb{E}}\left[H^{(g)}\left(V_{1} \mid \boldsymbol{Y}=\mathbf{y}\right)\right] .
\end{aligned}
$$

Define a typical set. The above expression allows us to only consider "typical" outputs $\boldsymbol{y}$ for the all-zero input while approximating $\mathbb{E}_{g \sim G}\left[H^{(g)}\left(V_{1} \mid \boldsymbol{Y}\right)\right]$. For the BSC case, we consider $\boldsymbol{y}$ to be typical when $|w t(\mathbf{y})-\ell p| \leq 2 \sqrt{\ell} \log \ell$. It is clear that if zero-vector is transmitted through the channel, the output will be a vector from $\mathcal{F}$ with high probability. It means that we do not lose too much in terms of accuracy if we restrict our attention only to this typical set, so the following lower suffice as a good lower bound on the expectation

$$
\begin{aligned}
\underset{g \sim G}{\mathbb{E}}\left[H^{(g)}\left(V_{1} \mid \boldsymbol{Y}\right)\right] & \\
& \geq \sum_{|w t(\mathbf{y})-\ell p| \leq 2 \sqrt{\ell} \log \ell} p^{w t(y)}(1-p)^{\ell-w t(y)} \underset{g \sim G}{\mathbb{E}}\left[H^{(g)}\left(V_{1} \mid \boldsymbol{Y}=\boldsymbol{y}\right)\right] .
\end{aligned}
$$

Fix a typical output. Let us fix any typical $y \in \boldsymbol{y}^{\ell}$ such that $|w t(\mathbf{y})-\ell p| \leq 2 \sqrt{\ell} \log \ell$, and show that $\mathbb{E}_{g \sim G}\left[H^{(g)}\left(V_{1} \mid \boldsymbol{Y}=\mathbf{y}\right)\right]$ is very close to 1 . To do this, we first notice that

$$
H^{(g)}\left(V_{1} \mid \boldsymbol{Y}=\boldsymbol{y}\right)=h\left(\frac{\mathbb{P}^{(g)}\left(V_{1}=0, \boldsymbol{Y}=\boldsymbol{y}\right)}{\mathbb{P}^{(g)}(\boldsymbol{Y}=\boldsymbol{y})}\right) .
$$

It suffices to show that the ratio of the probabilities inside the above equation is very close to $1 / 2$ w.h.p. We are going to prove this by showing that both denominator and numerator are highly concentrated around their respective means for $g \sim G$, and that the means have a ratio nearly $1 / 2$. 
Denote $\widetilde{\boldsymbol{V}}=\boldsymbol{V}^{[2: k]}$ to be bits 2 to $k$ of vector $\boldsymbol{V}$, and by $\widetilde{g}=$ $g[2: k]$ the matrix $g$ without its first row. Next we define the shifted weight distributions of the codebooks generated by $g$ and $\widetilde{g}$ :

$$
\begin{aligned}
& B_{g}(d, \boldsymbol{y}):=\left|\left\{\boldsymbol{v} \in\{0,1\}^{k} \backslash \mathbf{0} \quad: w t(\mathbf{v} g+\boldsymbol{y})=d\right\}\right|, \\
& \widetilde{B}_{g}(d, \boldsymbol{y}):=\left|\left\{\widetilde{\boldsymbol{v}} \in\{0,1\}^{k-1} \backslash \mathbf{0}: w t(\widetilde{\mathbf{v}} \widetilde{g}+\boldsymbol{y})=d\right\}\right| .
\end{aligned}
$$

Therefore,

$$
\begin{gathered}
\frac{\mathbb{P}^{(g)}\left(V_{1}=0, \boldsymbol{Y}=\boldsymbol{y}\right)}{\mathbb{P}^{(g)}(\boldsymbol{Y}=\boldsymbol{y})}=\frac{\sum_{\widetilde{\mathbf{u}}} \mathbb{P}^{(g)}\left(\boldsymbol{Y}=\boldsymbol{y} \mid V_{1}=0, \widetilde{\boldsymbol{V}}=\widetilde{\mathbf{u}}\right)}{\sum_{\boldsymbol{u}} \mathbb{P}^{(g)}(\boldsymbol{Y}=\boldsymbol{y} \mid \boldsymbol{V}=\boldsymbol{u})} \\
=\frac{p^{w t(\boldsymbol{y})}(1-p)^{\ell-w t(y)}+\sum_{d=0}^{\ell} \widetilde{B}_{g}(d, \boldsymbol{y}) p^{d}(1-p)^{\ell-d}}{p^{w t(y)}(1-p)^{\ell-w t(y)}+\sum_{d=0}^{\ell} B_{g}(d, \boldsymbol{y}) p^{d}(1-p)^{\ell-d}} .
\end{gathered}
$$

We will prove a concentration of the above expression around $1 / 2$, which will then imply that $H^{(g)}\left(V_{1} \mid \boldsymbol{Y}=\boldsymbol{y}\right)$ is close to 1 with high probability by (15). To do this, we will prove concentrations around means for both numerator and denominator of the above ratio. Since the following arguments work in exactly the same way, let us only consider the denominator for now.

By definition,

$$
B_{g}(d, y)=\sum_{\mathbf{v} \neq \mathbf{0}} \mathbb{1}[w t(\mathbf{v} g+\mathbf{y})=d]
$$

For the variance and the expectation and variance of each summand we have

$$
\underset{g \sim G}{\operatorname{Var}} \mathbb{1}[w t(\mathbf{v} g+\mathbf{y})=d] \leq \underset{g \sim G}{\mathbb{E}} \mathbb{1}[w t(\mathbf{v} g+\mathbf{y})=d]=\left(\begin{array}{l}
\ell \\
d
\end{array}\right) 2^{-\ell} .
$$

for any $\mathbf{v} \in\{0,1\}^{k} \backslash \mathbf{0}$. Clearly, the summands in (17) are pairwise independent. Therefore,

$$
\underset{g \sim G}{\operatorname{Var}}\left[B_{g}(d, y)\right] \leq \underset{g \sim G}{\mathbb{E}}\left[B_{g}(d, y)\right]=\left(2^{k}-1\right)\left(\begin{array}{l}
\ell \\
d
\end{array}\right) 2^{-\ell},
$$

and then

$$
\begin{aligned}
\underset{g \sim G}{\mathbb{E}}\left[\sum_{d=0}^{\ell} B_{g}(d, y) p^{d}(1-p)^{\ell-d}\right] \\
=\left(2^{k}-1\right) 2^{-\ell}\left(\sum_{d=0}^{\ell}\left(\begin{array}{l}
\ell \\
d
\end{array}\right) p^{d}(1-p)^{\ell-d}\right)=\left(2^{k}-1\right) 2^{-\ell} .
\end{aligned}
$$

Let us now show that $\sum_{d=0}^{\ell} B_{g}(d, y) p^{d}(1-p)^{\ell-d}$ is tightly concentrated around its mean for $g \sim G$. To do this, we split the range of $d$ into two parts: when $|d-\ell p|>6 \sqrt{\ell} \log \ell$, and when $|d-\ell p| \leq 6 \sqrt{\ell} \log \ell:$

$$
\begin{gathered}
\sum_{d=0}^{\ell} B_{g}(d, y) p^{d}(1-p)^{\ell-d}=\sum_{|d-\ell p|>6 \sqrt{\ell} \log \ell} B_{g}(d, y) p^{d}(1-p)^{\ell-d} \\
+\sum_{|d-\ell p| \leq 6 \sqrt{\ell} \log \ell} B_{g}(d, y) p^{d}(1-p)^{\ell-d} .
\end{gathered}
$$
Negligible part. Denote $Z_{g}(\mathbf{y})=\sum_{|d-\ell p|>6 \sqrt{\ell} \log \ell} B_{g}(d, y) p^{d}(1-p)^{\ell-d}$, and notice that

$$
\begin{aligned}
\underset{g \sim G}{\mathbb{E}}\left[Z_{g}(\mathbf{y})\right] & =\left(2^{k}-1\right) 2^{-\ell} \sum_{|d-\ell p|>6 \sqrt{\ell} \log \ell}\left(\begin{array}{l}
\ell \\
d
\end{array}\right) p^{d}(1-p)^{\ell-d} \\
& \leq\left(2^{k}-1\right) 2^{-\ell} \cdot \exp \left(-12 \log ^{2} \ell\right) \\
& \leq 2\left(2^{k}-1\right) 2^{-\ell} \cdot \ell^{-12 \log \ell},
\end{aligned}
$$

where the inequality is obtained via the Chernoff bound for binomial random variable. Then Markov's inequality gives $\mathbb{P}_{g \sim G}[Z \geq$ $\left.\underset{g \sim G}{\mathbb{E}}\left[Z_{g}(\boldsymbol{y})\right] \ell^{2 \log \ell}\right] \leq \ell^{-2 \log \ell}$, and so

$$
\mathbb{P}\left[Z_{g}(\mathbf{y})<2\left(2^{k}-1\right) 2^{-\ell} \ell^{-10 \ell \log \ell}\right] \geq 1-\ell^{-2 \log \ell} .
$$

Define the set

$$
\mathcal{G}_{1}:=\left\{g \in\{0,1\}^{k \times \ell}: Z_{g}(\boldsymbol{y})<2\left(2^{k}-1\right) 2^{-\ell} \ell^{-10 \ell \log \ell}\right\},
$$

and then $\underset{g \sim G}{\mathbb{P}}\left[g \in \mathcal{G}_{1}\right] \geq 1-\ell^{-2 \log \ell}$.

Substantial part. Now we deal with the part when $|d-\ell p| \leq$ $6 \sqrt{\ell} \log \ell$. For now, let us fix any $d$ in this interval, and use Chebyshev's inequality together with (18):

$$
\begin{aligned}
& \underset{g \sim G}{\mathbb{P}}\left[\left|B_{g}(d, \mathbf{y})-\mathbb{E}\left[B_{g}(d, \boldsymbol{y})\right]\right| \geq \ell^{-2 \log \ell} \mathbb{E}\left[B_{g}(d, \boldsymbol{y})\right]\right] \\
& \leq \frac{\operatorname{Var}\left[B_{g}(d, \boldsymbol{y})\right]}{\ell^{-4 \log \ell} \mathbb{E}^{2}\left[B_{g}(d, \mathbf{y})\right]} \leq \frac{\ell^{4 \log \ell}}{\underset{g \sim G}{\mathbb{E}}\left[B_{g}(d, \boldsymbol{y})\right]} \leq \ell^{4 \log \ell} \frac{2^{\ell-k+1}}{\left(\begin{array}{l}
\ell \\
d
\end{array}\right)} .
\end{aligned}
$$

We use the following bound on the binomial coefficients

Fact 5.3 ([18], Chapter 10, Lemma 7). For any integer $0 \leq d \leq \ell$,

$$
\frac{1}{\sqrt{2 \ell}} 2^{\ell h(d / \ell)} \leq\left(\begin{array}{l}
\ell \\
d
\end{array}\right) \leq 2^{\ell h(d / \ell)}
$$

Since we fixed $|d-\ell p| \leq 6 \sqrt{\ell} \log \ell$, Proposition 3.1 implies

$$
\begin{aligned}
\left|h(p)-h\left(\frac{d}{\ell}\right)\right| \leq h\left(6 \ell^{-1 / 2} \log \ell\right) & \leq 12 \ell^{-1 / 2} \log \ell \cdot \log \frac{\ell^{1 / 2}}{6 \log \ell} \\
& \leq 6 \ell^{-1 / 2} \log ^{2} \ell .
\end{aligned}
$$

Recalling that we consider the above-capacity regime with $k \geq \ell(1-h(p))+8 \sqrt{\ell} \log ^{2} \ell$, we derive from (21) and above

$$
\frac{2^{\ell-k+1}}{\left(\begin{array}{l}
\ell \\
d
\end{array}\right)} \leq \ell 2^{\ell\left[h(p)-h\left(\frac{d}{\ell}\right)-8 \ell^{-1 / 2} \log ^{2} \ell\right]} \leq \ell 2^{-2 \ell^{1 / 2} \log ^{2} \ell} .
$$

Therefore, we get in (20):

$$
\begin{aligned}
& \underset{g \sim G}{\mathbb{P}}\left[\left|B_{g}(d, \boldsymbol{y})-\mathbb{E}\left[B_{g}(d, y)\right]\right|\right.\left.\geq \ell^{-2 \log \ell} \mathbb{E}\left[B_{g}(d, \boldsymbol{y})\right]\right] \\
& \leq \ell^{4 \log \ell+1} 2^{-2 \ell^{1 / 2} \log ^{2} \ell} \leq \ell^{-\sqrt{\ell}-1} .
\end{aligned}
$$

Finally, denote

$$
\begin{array}{r}
\mathcal{G}_{2}:=\left\{g:\left|B_{g}(d, \mathbf{y})-\mathbb{E}\left[B_{g}(d, \mathbf{y})\right]\right| \leq \ell^{-2 \log \ell} \mathbb{E}\left[B_{g}(d, \boldsymbol{y})\right]\right. \\
\text { for all }|d-\ell p| \leq 6 \sqrt{\ell} \log \ell\} .
\end{array}
$$


Then by a simple union bound applied to (22) for all $d$ such that $|d-\ell p| \leq 6 \sqrt{\ell} \log \ell$ we obtain

$$
\underset{g \sim G}{\mathbb{P}}\left[g \in \mathcal{G}_{2}\right] \geq 1-\ell^{-\sqrt{\ell}} .
$$

We are now ready to combine these bounds to get the needed concentration.

Lemma 5.4. With probability at least $1-2 \ell^{-2 \log \ell}$ over the choice of $g \sim G$, it holds

$$
\begin{array}{r}
\left(2^{k}-1\right) 2^{-\ell}\left(1-2 \ell^{-2 \log \ell}\right) \leq \sum_{d=0}^{\ell} B_{g}(d, y) p^{d}(1-p)^{\ell-d} \\
\leq\left(2^{k}-1\right) 2^{-\ell}\left(1+2 \ell^{-2 \log \ell}\right) .
\end{array}
$$

Proof. Indeed, by union bound $\mathbb{P}_{g \sim G}\left[g \in \mathcal{G}_{1} \cap \mathcal{G}_{2}\right] \geq 1-$ $l^{-2 \log \ell}-\ell^{\sqrt{\ell}} \geq 1-2 \ell^{-2 \log \ell}$. But for any $g \in \mathcal{G}_{1} \cap \mathcal{G}_{2}$ we have from (23)

$$
\begin{aligned}
\sum_{d=0}^{\ell} B_{g}(d, \boldsymbol{y}) p^{d}(1-p)^{\ell-d} & \\
& \geq \sum_{|d-\ell p| \leq 6 \sqrt{\ell} \log \ell} B_{g}(d, \boldsymbol{y}) p^{d}(1-p)^{\ell-d} \\
& \geq\left(2^{k}-1\right) 2^{-\ell}\left(1-\ell^{-2 \log \ell}\right) \sum_{|d-\ell p| \leq 6 \sqrt{\ell} \log \ell}\left(\begin{array}{l}
\ell \\
d
\end{array}\right) p^{d}(1-p)^{\ell-d} \\
& \geq\left(2^{k}-1\right) 2^{-\ell}\left(1-\ell^{-2 \log \ell}\right)\left(1-2 \ell^{-12 \log \ell}\right) \\
& \geq\left(2^{k}-1\right) 2^{-\ell}\left(1-2 \ell^{-2 \log \ell}\right) .
\end{aligned}
$$

We can also upper bound using (23) and (19)

$$
\begin{aligned}
\sum_{d=0}^{\ell} & B_{g}(d, \boldsymbol{y}) p^{d}(1-p)^{\ell-d} \\
= & \sum_{|d-\ell p| \leq 6 \sqrt{\ell} \log \ell} B_{g}(d, \boldsymbol{y}) p^{d}(1-p)^{\ell-d} \\
& \quad+\sum_{|d-\ell p|>6 \sqrt{\ell} \log \ell} B_{g}(d, \mathbf{y}) p^{d}(1-p)^{\ell-d} \\
\leq & \left(2^{k}-1\right) 2^{-\ell}\left(1+\ell^{-2 \log \ell}\right) \sum_{|d-\ell p| \leq 6 \sqrt{\ell} \log \ell}\left(\begin{array}{l}
\ell \\
d
\end{array}\right) p^{d}(1-p)^{\ell-d}+Z_{g}(\mathbf{y}) \\
\leq & \left(2^{k}-1\right) 2^{-\ell}\left(1+\ell^{-2 \log \ell}\right)+2\left(2^{k}-1\right) 2^{-\ell} \ell^{-10 \log \ell} \\
\leq & \left(2^{k}-1\right) 2^{-\ell}\left(1+2 \ell^{-2 \log \ell}\right) .
\end{aligned}
$$

We similarly obtain the concentration for the sum in the numerator of (16): with probability at least $1-2 \ell^{-2 \log \ell}$ over the choice of $g$, it holds

$$
\begin{aligned}
\left(2^{k-1}-1\right) 2^{-\ell}\left(1-2 \ell^{-2 \log \ell}\right) \leq \sum_{d=0}^{\ell} & \widetilde{B}_{g}(d, y) p^{d}(1-p)^{\ell-d} \\
& \leq\left(2^{k-1}-1\right) 2^{-\ell}\left(1+2 \ell^{-2 \log \ell}\right) .
\end{aligned}
$$

Next, let us use the fact the we took a typical output $y$ with $|w t(y)-\ell p| \leq 2 \sqrt{\ell} \log \ell$ to show that the terms $p^{w t(y)}(1-$ $p)^{\ell-w t(y)}$ are negligible in both numerator and denominator of (16). We have

$$
\begin{aligned}
p^{w t(y)}(1-p)^{\ell-w t(y)} & =\left(\frac{1-p}{p}\right)^{\ell p-w t(y)} \cdot p^{\ell p}(1-p)^{\ell-\ell p} \\
& =2^{(\ell p-w t(y)) \cdot \log \left(\frac{1-p}{p}\right)} \cdot 2^{-\ell h(p)} .
\end{aligned}
$$

Simple case analysis gives us:

(a) If $p<\frac{1}{\sqrt{\ell}}$, then $(\ell p-w t(y)) \cdot \log \left(\frac{1-p}{p}\right) \leq \ell p \log \frac{1}{p}<$ $\ell \frac{1}{\sqrt{\ell}} \log \sqrt{\ell}<\sqrt{\ell} \log ^{2} \ell ;$

(b) In case $p \geq \frac{1}{\sqrt{\ell}}$, obtain $(\ell p-w t(y)) \cdot \log \left(\frac{1-p}{p}\right) \leq 2 \sqrt{\ell} \log \ell$. $\log \frac{1}{p} \leq \sqrt{\ell} \log ^{2} \ell$.

Using the above in (26) we derive for $k \geq \ell(1-h(p))+8 \sqrt{\ell} \log ^{2} \ell$ $p^{w t(y)}(1-p)^{\ell-w t(y)} \leq 2^{\sqrt{\ell} \log ^{2} \ell-\ell h(p)} \leq 2^{2 \sqrt{\ell} \log ^{2} \ell-\ell h(p)-2 \log ^{2} \ell-2}$

$$
\leq \ell^{-2 \log \ell}\left(2^{k-1}-1\right) 2^{-\ell} .
$$

Combining this with (24) and (25) and using a union bound we derive that with probability at least $1-4 \ell^{-2 \log \ell}$ it holds

$$
\begin{array}{r}
\left|\left(p^{w t(y)}(1-p)^{\ell-w t(y)}+\sum_{d=0}^{\ell} B_{g}(d, y) p^{d}(1-p)^{\ell-d}\right)-\left(2^{k}-1\right) 2^{-\ell}\right| \\
\leq 3 \ell^{-2 \log \ell} \cdot\left(2^{k}-1\right) 2^{-\ell}, \\
\left|\left(p^{w t(y)}(1-p)^{\ell-w t(y)}+\sum_{d=0}^{\ell} \widetilde{B}_{g}(d, y) p^{d}(1-p)^{\ell-d}\right)-\left(2^{k-1}-1\right) 2^{-\ell}\right| \\
\leq 3 \ell^{-2 \log \ell} \cdot\left(2^{k-1}-1\right) 2^{-\ell} .
\end{array}
$$

Therefore, with probability at least $1-4 \ell^{-2 \log \ell}$ the expression in (16) is bounded as

$$
\begin{aligned}
\frac{\left(1-3 \ell^{-2 \log \ell}\right)\left(2^{k-1}-1\right) 2^{-\ell}}{\left(1+3 \ell^{-2 \log \ell}\right)\left(2^{k}-1\right) 2^{-\ell}} \leq & \frac{\mathbb{P}^{(g)}\left(V_{1}=0, \boldsymbol{Y}=\boldsymbol{y}\right)}{\mathbb{P}^{(g)}(\boldsymbol{Y}=\boldsymbol{y})} \\
& \leq \frac{\left(1+3 \ell^{-2 \log \ell}\right)\left(2^{k-1}-1\right) 2^{-\ell}}{\left(1-3 \ell^{-2 \log \ell}\right)\left(2^{k}-1\right) 2^{-\ell}} .
\end{aligned}
$$

We can finally derive:

$$
\begin{gathered}
\frac{\left(1-3 \ell^{-2 \log \ell}\right)\left(2^{k-1}-1\right)}{\left(1+3 \ell^{-2 \log \ell}\right)\left(2^{k}-1\right)} \geq\left(1-6 \ell^{-2 \log \ell}\right)\left(\frac{1}{2}-2^{-k}\right) \\
\geq\left(1-6 \ell^{-2 \log \ell}\right)\left(\frac{1}{2}-\ell^{-8 \sqrt{\ell} \log \ell}\right) \geq \frac{1}{2}-\ell^{-\log \ell}, \\
\frac{\left(1+3 \ell^{-2 \log \ell}\right)\left(2^{k-1}-1\right)}{\left(1-3 \ell^{-2 \log \ell}\right)\left(2^{k}-1\right)} \leq\left(1+9 \ell^{-2 \log \ell}\right) \frac{1}{2} \leq \frac{1}{2}+\ell^{-\log \ell .}
\end{gathered}
$$

Therefore, with probability at least $1-4 \ell^{-2 \log \ell}$ over $g \sim G$ it holds

$$
\left|\frac{\mathbb{P}^{(g)}\left(V_{1}=0, \boldsymbol{Y}=\boldsymbol{y}\right)}{\mathbb{P}^{(g)}(\boldsymbol{Y}=\boldsymbol{y})}-\frac{1}{2}\right| \leq \ell^{-\log \ell} .
$$


Since $h(1 / 2+x) \geq 1-4 x^{2}$ for any $x \in(-1 / 2,1 / 2)$, we then derive:

$$
\begin{aligned}
\underset{g \sim G}{\mathbb{E}}\left[H ^ { ( g ) } \left(V_{1} \mid \boldsymbol{Y}\right.\right. & =\boldsymbol{y})]=\underset{g \sim G}{\mathbb{E}}\left[h\left(\frac{\mathbb{P}^{(g)}\left(V_{1}=0, \boldsymbol{Y}=\boldsymbol{y}\right)}{\mathbb{P}^{(g)}(\boldsymbol{Y}=\boldsymbol{y})}\right)\right] \\
& \geq\left(1-4 \ell^{-2 \log \ell}\right)\left(1-4 \ell^{-2 \log \ell}\right) \geq 1-8 \ell^{-2 \log \ell} .
\end{aligned}
$$

Concentration of entropy. We are now ready to plug this into (14):

$$
\begin{aligned}
& \underset{g \sim G}{\mathbb{E}}\left[H^{(g)}\left(V_{1} \mid \boldsymbol{Y}\right)\right] \\
& \geq\left(1-8 \ell^{-2 \log \ell}\right) \sum_{|w t(\mathbf{y})-\ell p| \leq 2 \sqrt{\ell} \log \ell} p^{w t(y)}(1-p)^{\ell-w t(y)} \\
&=\left(1-8 \ell^{-2 \log \ell}\right) \sum_{|d-\ell p| \leq 2 \sqrt{\ell} \log \ell}\left(\begin{array}{l}
\ell \\
d
\end{array}\right) p^{d}(1-p)^{\ell-d} \\
& \geq\left(1-8 \ell^{-2 \log \ell}\right)\left(1-2 \ell^{-2 \log \ell}\right) \\
& \geq 1-10 \ell^{-2 \log \ell} .
\end{aligned}
$$

Finally, using the fact that $H^{(g)}\left(V_{1} \mid \boldsymbol{Y}\right) \leq 1$, Markov's inequality, and (27), we get

$$
\begin{gathered}
\underset{g \sim G}{\mathbb{P}}\left[H^{(g)}\left(V_{1} \mid \boldsymbol{Y}\right) \leq 1-\ell^{-\log \ell}\right]=\underset{g \sim G}{\mathbb{P}}\left[1-H^{(g)}\left(V_{1} \mid \boldsymbol{Y}\right) \geq \ell^{-\log \ell}\right] \\
\leq \frac{\underset{g \sim G}{\mathbb{E}}\left[1-H^{(g)}\left(V_{1} \mid \boldsymbol{Y}\right)\right]}{\ell^{-\log \ell}} \leq 10 \ell^{-\log \ell} .
\end{gathered}
$$

Thus we conclude that with probability at least $1-10 \ell^{-\log \ell}$ over the choice of the kernel $G$ it holds that $H\left(V_{1} \mid \boldsymbol{Y}\right) \geq 1-\ell^{-\log \ell}$ when $k \geq \ell(1-h(p))+8 \sqrt{\ell} \log ^{2} \ell$ and the underlying channel is BSC. This completes the proof of Theorem 5.1, which is a version of Theorem 4.7 for the BSC case.

\section{ACKNOWLEDGMENTS}

We are grateful to Hamed Hassani for useful discussions and sharing his insights on random coding theorems during the initial stages of this work.

The first two authors were supported in part by NSF grants CCF1422045 and CCF-1563742. The first author was supported in part by a Google Research Award. Some of this research was carried out when the third author was visiting Carnegie Mellon University.

\section{REFERENCES}

[1] Erdal Arıkan. 2009. Channel Polarization: A method for constructing capacityachieving codes for symmetric binary-input memoryless channels. IEEE Transactions on Information Theory (July 2009), 3051-3073.

[2] Erdal Arıkan and Emre Telatar. 2009. On the Rate of Channel Polarization. In Proceedings of 2009 IEEE International Symposium on Information Theory. 14931495.

[3] Alexander Barg and G. David Forney. 2002. Random codes: minimum distances and error exponents. IEEE Transactions on Information Theory 48, 9 (Sep. 2002), 2568-2573. https://doi.org/10.1109/TIT.2002.800480

[4] M. Benammar, V. Bioglio, F. Gabry, and I. Land. 2017. Multi-kernel polar codes: Proof of polarization and error exponents. In 2017 IEEE Information Theory Workshop (ITW). IEEE, 101-105.

[5] Jaroslaw Blasiok, Venkatesan Guruswami, Preetum Nakkiran, Atri Rudra, and Madhu Sudan. 2018. General strong polarization. In Proceedings of the 50th Annual ACM SIGACT Symposium on Theory of Computing. ACM, 485-492.
[6] Y. Domb, R. Zamir, and M. Feder. 2016. The Random Coding Bound Is Tight for the Average Linear Code or Lattice. IEEE Transactions on Information Theory 62, 1 (Jan 2016), 121-130. https://doi.org/10.1109/TIT.2015.2496308

[7] Arman Fazeli, S. Hamed Hassani, Marco Mondelli, and Alexander Vardy. 2017. Binary Linear Codes with Optimal Scaling and Quasi-Linear Complexity. ArXiv e-prints (Nov. 2017). arXiv:cs.IT/1711.01339

[8] Arman Fazeli and Alexander Vardy. 2014. On the scaling exponent of binary polarization kernels. In 2014 52nd Annual Allerton Conference on Communication, Control, and Computing (Allerton). 797-804. https://doi.org/10.1109/ALLERTON. 2014.7028536

[9] G. David Forney. 1967. Concatenated codes. Ph.D. Dissertation. Massachusetts Institute of Technology

[10] G. David Forney. 2005. On exponential error bounds for random codes on the BSC. Lecture notes (2005). Available at http://web.mit.edu/6.441/spring05/reading/Forney_ExpEBBSC.pdf.

[11] F. Gabry, V. Bioglio, I. Land, and J. Belfiore. 2017. Multi-kernel construction of polar codes. In 2017 IEEE International Conference on Communications Workshops (ICC Workshops). IEEE, 761-765.

[12] R. Gallager. 1965. A simple derivation of the coding theorem and some applications. IEEE Transactions on Information Theory 11, 1 (January 1965), 3-18. https://doi.org/10.1109/TIT.1965.1053730

[13] Dina Goldin and David Burshtein. 2014. Improved Bounds on the Finite Length Scaling of Polar Codes. IEEE Trans. Information Theory 60, 11 (2014), 6966-6978.

[14] Venkatesan Guruswami and Patrick Xia. 2015. Polar Codes: Speed of Polarization and Polynomial Gap to Capacity. IEEE Trans. Information Theory 61, 1 (2015), 3-16. Preliminary version in Proc. of FOCS 2013.

[15] S. H. Hassani, K. Alishahi, and R. L. Urbanke. 2014. Finite-Length Scaling for Polar Codes. IEEE Transactions on Information Theory 60, 10 (Oct 2014), 5875-5898. https://doi.org/10.1109/TIT.2014.2341919

[16] A. G. i. Fabregas, I. Land, and A. Martinez. 2011. Extremes of random coding error exponents. In 2011 IEEE International Symposium on Information Theory Proceedings. 2896-2898. https://doi.org/10.1109/ISIT.2011.6034105

[17] Satish Babu Korada, Eren Sasoglu, and Rüdiger L. Urbanke. 2010. Polar Codes: Characterization of Exponent, Bounds, and Constructions. IEEE Transactions on Information Theory 56, 12 (2010), 6253-6264.

[18] Florence Jessie MacWilliams and Neil James Alexander Sloane. 1977. The theory of error-correcting codes. Vol. 16. Elsevier.

[19] Vera Miloslavskaya and Peter Trifonov. 2012. Design of binary polar codes with arbitrary kernel. 2012 IEEE Information Theory Workshop (2012), 119-123.

[20] Marco Mondelli, S. Hamed Hassani, and Rüdiger L. Urbanke. 2016. Unified Scaling of Polar Codes: Error Exponent, Scaling Exponent, Moderate Deviations, and Error Floors. IEEE Trans. Information Theory 62, 12 (2016), 6698-6712. https: //doi.org/10.1109/TIT.2016.2616117

[21] Ryuhei Mori and Toshiyuki Tanaka. 2014. Source and Channel Polarization Over Finite Fields and Reed-Solomon Matrices. IEEE Trans. Information Theory 60, 5 (2014), 2720-2736.

22] Henry D. Pfister and Rüdiger L. Urbanke. 2016. Near-optimal finite-length scaling for polar codes over large alphabets. In IEEE International Symposium on Information Theory, ISIT. 215-219.

[23] Yury Polyanskiy, H Vincent Poor, and Sergio Verdú. 2010. Channel coding rate in the finite blocklength regime. IEEE Transactions on Information Theory 56, 5 (2010), 2307.

[24] Noam Presman, Ofer Shapira, and Simon Litsyn. 2015. Mixed-kernels constructions of polar codes. IEEE fournal on Selected Areas in Communications 34, 2 (2015), 239-253.

[25] Claude Elwood Shannon. 1948. A mathematical theory of communication. Bell system technical journal 27, 3 (1948), 379-423.

[26] Volker Strassen. 1962. Asymptotische Abschatzungen in Shannon's Informationstheories. In Trans. 3rd Prague Conf. Info. Theory. 689-723.

[27] Volker Strassen. 2009. Asymptotic estimates in Shannon's information theory. In Proc. Trans. 3rd Prague Conf. Inf. Theory. 689-723.

[28] Ido Tal and Alexander Vardy. 2013. How to Construct Polar Codes. IEEE Transactions on Information Theory 59, 10 (Oct 2013), 6562-6582.

[29] Hsin-Po Wang and Iwan Duursma. 2018. Polar-like Codes and Asymptotic Tradeoff among Block Length, Code Rate, and Error Probability. (2018). arXiv: 1812.08112

[30] Hsin-Po Wang and Iwan M. Duursma. 2019. Polar Codes' Simplicity, Random Codes' Durability. ArXiv abs/1912.08995 (2019).

[31] Jacob Wolfowitz. 1957. The coding of messages subject to chance errors. Illinois 7. Math. 1 (1957), 591-606.

[32] Hanwen Yao, Arman Fazeli, and Alexander Vardy. 2019. Explicit Polar Codes with Small Scaling Exponent. In 2019 IEEE International Symposium on Information Theory (ISIT). 1757-1761. https://doi.org/10.1109/ISIT.2019.8849741

[33] Min Ye and Alexander Barg. 2015. Polar codes using dynamic kernels. In 2015 IEEE International Symposium on Information Theory (ISIT). IEEE, 231-235. 